УДК 004.8

\title{
Formalisms for conceptual design of information systems*
}

\author{
Anureev I.S. (Institute of Informatics Systems)
}

A class of information systems considered in this paper is defined as follows: a system belongs to the class if its change can be caused by both its environment and factors inside the system, and there is an information transfer from it to its environment and from its environment to it. Two formalisms (information transition systems and conceptual transition systems) for abstract unified modelling of the artifacts (concept sketches and models) of the conceptual design of information systems of the class, early phase of information systems design process, are proposed. Information transition defines the abstract unified information model for the artifacts, based on such general concepts as state, information query, answer and transition. Conceptual transition systems are a formalism for conceptual modelling of information transition systems. They defines the abstract unified conceptual model for the artifacts. The basic definitions of the theory of conceptual transition systems are given. A language of conceptual transition systems is defined.

Keywords: information system, information transition system, conceptual structure, ontology, ontological element, conceptual, conceptual state, conceptual configuration, conceptual transition system, conceptual information transition model, transition system, CTSL

\section{Introduction}

The conceptual models play an important role in the overall system development life cycle [1]. Numerous conceptual modelling techniques have been created, but all of them have a limited number of kinds of ontological elements and therefore can only represent ontological elements of fixed conceptual granularity. For example, entity-relationship modelling technique [2] uses two kinds of ontological elements: entities and relationships.

The purpose of the paper is propose formalisms for abstract unified modelling of the artifacts (concept sketches and models) of the conceptual design of information systems (IS for short) by ontological elements of arbitrary conceptual granularity. In our two stage approach the informational and conceptual aspects of the system that the conceptual model represents are described by two separate formalisms. The first formalism describes the informational model of the system, and the second formalism describes the conceptual model of the informational

Partially supported by RFBR under grants 15-01-05974 and 15-07-04144 and SB RAS interdisciplinary integration project No.15/10. 
model.

An information transition system (ITS for short) is an extension of an information query system (IQS for short) characterized additionally by the exogenous and endogenous transition relations specifying transitions on states. The exogenous transition relation models change of an information system caused by its environment. It associates queries with binary relations on states called transition relations and answers returning by state pairs from these transition relations called transitions. The endogenous transition relation models change of an information system caused by factors inside the system. It is defined as a transition relation with answers returning by transitions of the transition relation.

A wide variety of information systems is modelled by ITSs in the information aspect, including database management systems with transitions initiated by queries, expert systems with transitions initiated by operations with facts and rules, social networks with transitions initiated by actions of users in accordance with certain communications protocols, abstract machines specifying operational semantics of programming languages with transitions initiated by instructions of abstract machines, verification condition generators specifying axiomatic semantics of programming languages with transitions initiated by inference rules and so on.

We consider that the second formalism used for for conceptual modelling of ITSs must meet the following general requirements (in relation to modelling of a ITS):

1. It must model the conceptual structure of states and state objects of the ITS.

2. It must model the content of the conceptual structure.

3. It must model information queries, information query objects, answers and answer objects of the IQS.

4. It must model the interpretation function of the ITS.

5. It must be quite universal to model typical ontological elements (concepts, attributes, concept instances, relations, relation instances, individuals, types, domains, and so on.).

6. It must provide a quite complete classification of ontological elements, including the determination of their new kinds and subkinds with arbitrary conceptual granularity.

7. The model of the interpretation function must be extensible.

8. It must have language support. The language associated with the formalism must define syntactic representations of models of states, state objects, queries, query objects, answers and answer objects and includes the set of predefined basic query models.

9. It must model the change of the conceptual structure of states and state objects of the 
ITS.

10. It must model the change of the content of the conceptual structure.

11. It must model the transition relations of the ITS.

12. The model of the exogenous transition relation must be extensible.

As is shown in [3], conceptual configuration systems (CCSs for short) meet the seven requirements in relation to IQSs. Comparison of CCSs with the abstract state machines $[4,5]$ which partially meet these requirements was made in [3]. In this paper we present an extension of CCSs, conceptual transition systems (CTSs for short) as the formalism satisfying the all above requirements.

The paper has the following structure. The preliminary concepts and notation are given in section 2. The basic definitions of the theory of CTSs are given in section 3. The language CTSL of CTSs is described in section 4. Semantics of executable elements in CTSL is defined in 5. We establish that CTSs meet the above requirements in section 6 .

\section{Preliminaries}

\subsection{Sets, sequences, multisets}

Let $O_{b}$ be the set of objects considered in this paper. Let $S_{t}$ be a set of sets. Let $I_{n t}, N_{t}$, $N_{t 0}$ and $B_{l}$ be sets of integers, natural numbers, natural numbers with zero and boolean values true and false, respectively.

Let the names of sets be represented by capital letters possibly with subscripts and the elements of sets be represented by the corresponding small letters possibly with extended subscripts. For example, $i_{n t}$ and $i_{n t .1}$ are elements of $I_{n t}$.

Let $S_{q}$ be a set of sequences. Let $s_{t .(*)}, s_{t .\{*\}}$, and $s_{t * *}$ denote sets of sequences of the forms $\left(o_{b .1}, \ldots, o_{b . n_{t 0}}\right),\left\{o_{b .1}, \ldots, o_{b . n_{t 0}}\right\}$, and $o_{b .1}, \ldots, o_{b . n_{t 0}}$ from elements of $s_{t}$. For example, $I_{n t .(*)}$ is a set of sequences of the form $\left(i_{n t .1}, \ldots, i_{n t . n t 0}\right)$, and $i_{n t . *}$ is a sequence of the form $i_{n t .1}, \ldots, i_{n t . n_{t 0}}$. Let $o_{b .1}, \ldots, o_{b . n_{t 0}}$, denote $o_{b .1}, \ldots, o_{b . n_{t 0}}$. Let $s_{t .\left(* n_{t 0}\right)}, s_{t .\left\{* n_{t 0}\right\}}$, and $s_{t . * n_{t 0}}$ denote sets of the corresponding sequences of the length $n_{t 0}$.

Let $o_{b .1} \prec_{\llbracket s_{q} \rrbracket} o_{b .2}$ denote the fact that there exist $o_{b . * .1}, o_{b . * .2}$ and $o_{b . * .3}$ such that $s_{q}=$

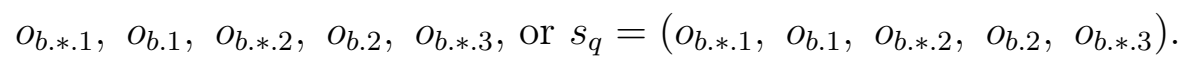

Let $\left[o_{b} O_{b .1} \hookleftarrow o_{b .2}\right]$ denote the result of replacement of all occurrences of $o_{b .1}$ in $o_{b}$ by $o_{b .2}$. Let $\left[s_{q} o_{b} \hookleftarrow_{*} O_{b .1}\right]$ denote the result of replacement of each element $o_{b .2}$ in $s_{q}$ by $\left[o_{b .1} o_{b} \hookleftarrow o_{b .2}\right]$. For example, $\left[(a, b) x \hookleftarrow_{*}(f x)\right]$ denotes $((f a),(f b))$. 
Let $\left[\right.$ len $\left.s_{q}\right]$ denote the length of $s_{q}$. Let und denote the undefined value. Let $\left[s_{q} \cdot n_{t}\right]$ denote the $n_{t}$ th element of $s_{q}$. If $\left[\right.$ len $\left.s_{q}\right]<n_{t}$, then $\left[s_{q} \cdot n_{t}\right]=$ und. Let $\left[s_{q}+s_{q .1}\right],\left[o_{b} \cdot+s_{q}\right]$ and $\left[s_{q}+. o_{b}\right]$ denote $o_{b . *}, o_{b . * 1}, o_{b}, o_{b . *}$ and $o_{b . *}, o_{b}$, where $s_{q}=o_{b . *}$ and $s_{q .1}=o_{b . * .1}$.

Let $\left[\right.$ and $\left.s_{q}\right]$ denote $\left(c_{n d .1}\right.$ and $\ldots$ and $\left.c_{n d . n_{t}}\right)$, where $s_{q}=c_{n d .1}, \ldots, c_{n d . n_{t}}$, and $[$ and $]$ denote true. In the case of $n_{t}=1$, the brackets can be omitted.

Let $o_{b .1}, o_{b .2} \in S_{t} \cup S_{q}$. Then $o_{b .1}={ }_{s t} o_{b .2}$ denote that the sets of elements of $o_{b .1}$ and $o_{b .2}$ coincide, and $o_{b .1}={ }_{m l} o_{b .2}$ denote that the multisets of elements of $o_{b .1}$ and $o_{b .2}$ coincide.

\subsection{Contexts}

The terms used in the paper are context-dependent.

Let $L_{b}$ be a set of objects called labels. Contexts have the form $\llbracket o_{b . *} \rrbracket$, where the elements of $o_{b . *}$ called embedded contexts have the form: $l_{b}: o_{b}, l_{b}$ : or $o_{b}$.

The context in which some embedded contexts are omitted is called a partial context. All omitted embedded contexts are considered bound by the existential quantifier, unless otherwise specified.

Let $o_{b} \llbracket o_{b . *} \rrbracket$ denote the object $o_{b}$ in the context $\llbracket o_{b . *} \rrbracket$.

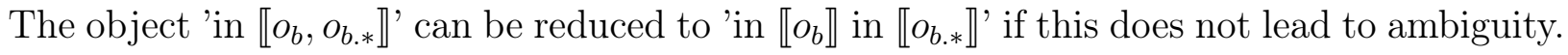

\subsection{Functions}

Let $F_{n}$ be a set of functions. Let $A_{r g}$ and $V_{l}$ be sets of objects called arguments and values. Let $\left[\begin{array}{ll}f_{n} & a_{r g * *}\end{array}\right]$ denote the application of $f_{n}$ to $a_{r g . *}$.

Let $\left[\right.$ support $\left.f_{n}\right]$ denote the support in $\llbracket f_{n} \rrbracket$, i. e. $\left[\right.$ support $\left.f_{n}\right]=\left\{a_{r g}:\left[f_{n} a_{r g}\right] \neq\right.$ und $\}$. Let $\left[\right.$ image $\left.f_{n} s_{t}\right]$ denote the image in $\llbracket f_{n}, s_{t} \rrbracket$, i. e. [image $\left.f_{n} s_{t}\right]=\left\{\left[\begin{array}{ll}f_{n} & a_{r g}\end{array}\right]: a_{r g} \in s_{t}\right\}$. Let [image $\left.f_{n}\right]$ denote the image in $\llbracket f_{n},\left[\right.$ support $\left.f_{n}\right] \rrbracket$. Let $\left[\right.$ narrow $\left.f_{n} s_{t}\right]$ denote the function $f_{n .1}$ such that $\left[\right.$ support $\left.f_{n .1}\right]=\left[\right.$ support $\left.f_{n .1}\right] \cap s_{t}$, and $\left[f_{n .1} a_{r g}\right]=\left[f_{n} a_{r g}\right]$ for each $a_{r g} \in\left[\right.$ support $\left.f_{n .1}\right]$. The function $f_{n .1}$ is called a narrowing of $f_{n}$ to $s_{t}$. Let $\left[\right.$ support $\left.f_{n .1}\right] \cap\left[\right.$ support $\left.f_{n .2}\right]=\emptyset$. Let $f_{n .1} \cup f_{n .2}$ denote the union $f_{n}$ of $f_{n .1}$ and $f_{n .2}$ such that $\left[\begin{array}{ll}f_{n} & a_{r g}\end{array}\right]=\left[\begin{array}{ll}f_{n .1} & a_{r g}\end{array}\right]$ for each $a_{r g} \in$ [support $\left.f_{n .1}\right]$, and $\left[f_{n} a_{r g}\right]=\left[\begin{array}{ll}f_{n .2} & a_{r g}\end{array}\right]$ for each $a_{r g} \in\left[\right.$ support $\left.f_{n .2}\right]$. Let $f_{n .1} \subseteq f_{n .2}$ denote the fact that $\left[\right.$ support $\left.f_{n .1}\right] \subseteq\left[\right.$ support $\left.f_{n .2}\right]$, and $\left[f_{n .1} a_{r g}\right]=\left[\begin{array}{lll}f_{n .2} & a_{r g}\end{array}\right]$ for each $a_{r g} \in\left[\operatorname{support} f_{n .1}\right]$.

An object $u_{p}$ of the form $a_{r g}: v_{l}$ is called an update. Let $U_{p}$ be a set of updates. The objects $a_{r g}$ and $v_{l}$ are called an argument and value in $\llbracket u_{p} \rrbracket$.

Let $\left[\begin{array}{ll}f_{n} & u_{p}\end{array}\right]$ denote the function $f_{n .1}$ such that $\left[\begin{array}{ll}f_{n .1} & a_{r g}\end{array}\right]=\left[\begin{array}{ll}f_{n} & a_{r g}\end{array}\right]$ if $a_{r g} \neq a_{r g} \llbracket u_{p} \rrbracket$, and 


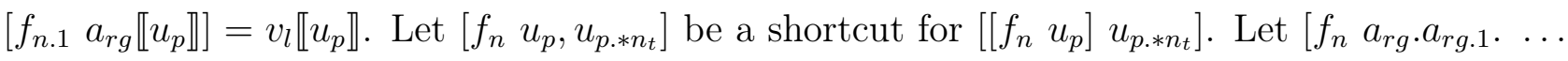
$\left.. a_{r g . n_{t}}: v_{l}\right]$ be a shortcut for $\left[f_{n} a_{r g}:\left[\left[\begin{array}{lll}f_{n} & a_{r g}\end{array}\right] a_{r g .1} \ldots \ldots . a_{r g . n_{t}}: v_{l}\right]\right]$. Let $\left[u_{p . *}\right]$ be a shortcut for $\left[\begin{array}{ll}f_{n} & u_{p . *}\end{array}\right]$, where $\left[\right.$ support $\left.f_{n}\right]=\emptyset$.

Let $C_{n d}$ be a set of objects called conditions. Let [if $c_{n d}$ then $o_{b .1}$ else $\left.o_{b .2}\right]$ denote the object $o_{b}$ such that

- if $c_{n d}=$ true, then $o_{b}=o_{b .1}$

- if $c_{n d}=$ false, then $o_{b}=o_{b .2}$.

\subsection{Attributes and multi-attributes}

An object $o_{b . m a}$ of the form $\left(u_{p . *}\right)$ is called a multi-attribute object. Let $O_{b . m a}$ be a set of multi-attribute objects. The elements of $\left[o_{b . m a} w \hookleftarrow_{*} a_{r g} \llbracket w \rrbracket\right]$ are called multi-attributes in $\llbracket o_{b . m a} \rrbracket$. Let $O_{b . m a}$ be a set of multi-attributes. The elements of $\left[o_{b . m a} w \hookleftarrow_{*} v_{l} \llbracket w \rrbracket\right]$ are

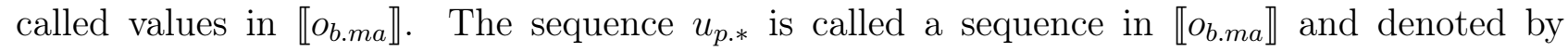
[sequence in $\left.o_{b . m a}\right]$. An object $v_{l}$ is a value in $\llbracket a_{t t . m}, o_{b . m a} \rrbracket$ if $o_{b . m a}=\left(u_{p . * .1}, a_{t t . m}: v_{l}, u_{p . * 2}\right)$ for some $u_{p . * .1}$ and $u_{p * * 2}$.

An object $o_{b . m a}$ is an attribute object if the elements of $\left[o_{b . m a} w \hookleftarrow_{*} a_{r g} \llbracket w \rrbracket\right]$ are pairwise distinct. Let $O_{b . a}$ be a set of attribute objects. The multi-attributes in $\llbracket o_{b . a} \rrbracket$ are called attributes in $\llbracket o_{b . a} \rrbracket$. Let $A_{t t}$ be a set of objects called attributes.

Let $\left[\right.$ function $\left.o_{b . a}\right],\left[o_{b . a} a_{t t}\right]$, and [support $\left.o_{b . a}\right]$ denote $\left[\left[\right.\right.$ sequence in $\left.\left.o_{b . a}\right]\right]$, [[function $\left.\left.o_{b . a}\right] a_{t t}\right]$, and [support [function $\left.\left.o_{b . a}\right]\right]$.

Let $\left[\right.$ seq-to-att-obj $\left.s_{q}\right]$ denote $\left(1:\left[s_{q} \cdot 1\right], \ldots,\left[\right.\right.$ len $\left.s_{q}\right]:\left[s_{q} \cdot\left[\right.\right.$ len $\left.\left.\left.s_{q}\right]\right]\right)$. Let $o_{b . a}=_{s t}(1$ : $\left.v_{l .1}, \ldots, n_{t}: v_{l . n_{t}}\right)$. Then $\left[a t t-o b j-t o-s e q o_{b . a}\right]$ denote $\left(v_{l .1}, \ldots, v_{l . n_{t}}\right)$.

\section{Basic definitions of the theory of conceptual transition systems}

Conceptual transition systems (CTSs) are transition systems in which states are conceptual configurations, and transition relations are binary relations on conceptual configurations. In this section the basic definitions of the theory of conceptual transition systems are presented. The defined structures of CTSs are constructed from atoms and, thus, defined implicitly in $\llbracket A_{t m} \rrbracket$.

\subsection{Information transition systems}


Let $S_{t t}$ be a set of objects called states. An element $t_{r n}$ of the form $\left(s_{t t .1}, s_{t t .2}\right)$ is called a transition. Let $T_{r n}$ be a set of transitions. The states $s_{t t .1}$ and $s_{t t .2}$ are called input and output states in $\llbracket t_{r n} \rrbracket$.

Let $S_{s . q}$ be a set of query systems. An object $s_{\text {s.t.i }}$ of the form $\left(s_{s . q}, t_{r n . r l t . e x}, t_{r n . r l t . e n}\right)$ is an information transition system if $t_{r n . r l t . e x} \in Q_{r} \times A_{n s} \times S_{t t} \times S_{t t} \rightarrow B_{l}, t_{r n . r l t . e n} \in A_{n s} \times S_{t t} \times S_{t t} \rightarrow$ $B_{l}$, and for all $q_{r} \in Q_{r}$ there exists $s_{t t} \in S_{t t}$ such that [value $\left.q_{r} s_{t t}\right] \neq$ und, or there exist $s_{t t .1} \in S_{t t}, s_{t t .2} \in S_{t t}$ and $a_{n s} \in A_{n s}$ such that $\left[\begin{array}{lllll}t_{r n . r l t . e x} & q_{r} & a_{n s} & s_{t t .1} & s_{t t .2}\end{array}\right]=t r u e$. Let $S_{s . t . i}$ be a set of information transition systems.

The system $s_{s . q}$ is called a query system in $\llbracket s_{s . t . i} \rrbracket$. The function $t_{r n . r l t . e x}$ is called an exogenous transition relation in $\llbracket s_{s . t . i} \rrbracket$. The function $t_{\text {rn.rlt.en }}$ is called an endogenous transition relation in $\llbracket s_{s . t . i} \rrbracket$. Let $s_{t t .1} \rightarrow_{q_{r}, a_{n s}} s_{t t .2}$ and $s_{t t .1} \rightarrow_{a_{n s}} s_{t t .2}$ be shortcuts for $\left[\begin{array}{llll}t_{r n . r l t . e x} & q_{r} a_{n s} s_{t t .1} s_{t t .2}\end{array}\right]=t r u e$ and $\left[\begin{array}{llll}t_{r n . r l t . e n} & a_{n s} & s_{t t .1} & s_{t t .2}\end{array}\right]=$ true, respectively.

The elements of $S_{t t} \llbracket s_{s . q} \rrbracket, O_{b . s} \llbracket s_{s . q} \rrbracket, Q_{r} \llbracket s_{s . q} \rrbracket, O_{b . q} \llbracket s_{s . q} \rrbracket, A_{n s} \llbracket s_{s . q} \rrbracket$ and $O_{b . a} \llbracket s_{s . q} \rrbracket$ are called states, state objects, queries, query objects, answers and answer objects in $\llbracket s_{s . t . i} \rrbracket$, respectively. The function value $\llbracket s_{s . q} \rrbracket$ is called a query interpretation in $\llbracket s_{\text {s.t.i. }} \rrbracket$.

A query $q_{r}$ is an information query in $\llbracket s_{s . t . i} \rrbracket$ if $\left[\right.$ value $\left.q_{r} s_{t t}\right] \neq$ und for some $s_{t t}$. A query $q_{r}$ is a change query in $\llbracket s_{s . t . i} \rrbracket$ if $\left[\begin{array}{lllll}t_{r n . r l t . e x} & q_{r} & a_{n s} & s_{t t .1} & s_{t t .2}\end{array}\right]=$ true for some $s_{t t .1}, s_{t t .2}$ and $a_{n s}$.

A system $s_{s . t . i}$ executes $t_{r n}$ if $s_{t t .1} \llbracket t_{r n} \rrbracket \rightarrow_{q_{r}, a_{n s}} s_{t t .2} \llbracket t_{r n} \rrbracket$ for some $q_{r}$ and $a_{n s}$, or $s_{t t .1} \llbracket t_{r n} \rrbracket \rightarrow a_{a_{n s}}$ $s_{t t .2} \llbracket t_{r n} \rrbracket$ for some $a_{n s}$. A system $s_{s . t . i}$ transits from $s_{t t .1}$ to $s_{t t .2}$ if $s_{s . t . i}$ executes $\left(s_{t t .1}, s_{t t .2}\right)$.

\subsection{Substitutions, patterns, pattern specifications, instances}

A function $s_{b} \in E_{l} \rightarrow E_{l . *}$ is called a substitution. Let $S_{b}$ be a set of substitutions. A function subst $\in S_{b} \times E_{l . *} \rightarrow E_{l . *}$ is a substitution function if it is defined as follows (the first proper rule is applied):

- if $e_{l} \in\left[\right.$ support $\left.s_{b}\right]$, then $\left[\right.$ subst $\left.s_{b} e_{l}\right]=\left[s_{b} e_{l}\right]$;

- $\left[\begin{array}{lll}\text { subst } s_{b} & a_{t m}\end{array}\right]=a_{t m}$;

- $\left[\right.$ subst $\left.s_{b} l_{b}: e_{l}\right]=\left[\right.$ subst $\left.s_{b} l_{b}\right]:\left[\right.$ subst $\left.s_{b} e_{l}\right]$;

- $\left[\right.$ subst $s_{b} e_{l}::$ nosubst $]=e_{l}$;

- $\left[\right.$ subst $s_{b} e_{l}::\left(\right.$ nosubstexcept $\left.\left.e_{l . *}\right)\right]=\left[\right.$ subst $\left[\right.$ narrow $\left.\left.s_{b}\left\{e_{l . *}\right\}\right] e_{l}\right]$;

- $\left[\right.$ subst $\left.s_{b} e_{l}:: s_{r t}\right]=\left[\right.$ subst $\left.s_{b} e_{l}\right]::\left[\right.$ subst $\left.s_{b} s_{r t}\right]$;

- $\left[\right.$ subst $\left.s_{b}\left(e_{l \cdot *}\right)\right]=\left(\left[e_{l . *} w \hookleftarrow_{*}\left[\right.\right.\right.$ subst $\left.\left.\left.s_{b} w\right]\right]\right)$;

- $\left[\right.$ subst $\left.s_{b} e_{l . *}\right]=\left[e_{l, *} w \hookleftarrow_{*}\left[\right.\right.$ subst $\left.\left.s_{b} w\right]\right]$. 
The sort nosubst specifies the elements to which the substitution $s_{b}$ is not applied. The sort (nosubstexcept $\left.e_{l . *}\right)$ specifies the elements to which the narrowing of the substitution $s_{b}$ to the set $e_{l . *}$ is applied. An element $p_{t}$ is a pattern in $\llbracket e_{l}, s_{b} \rrbracket$ if $\left[s u b s t s_{b} p_{t}\right]=e_{l}$. Let $P_{t}$ be a set of patterns. An element $i_{n s t}$ is an instance in $\llbracket p_{t}, s_{b} \rrbracket$ if $\left[\right.$ subst $\left.s_{b} p_{t}\right]=i_{n s t}$. Let $I_{n s t}$ be a set of instances.

Let $V_{r}$ and $V_{r . s}$ be sets of objects called element variables and sequence variables, respectively. An element $p_{t . s}$ of the form $\left(p_{t},\left(v_{r . *}\right),\left(v_{r . s . *}\right)\right)$ is a pattern specification if $\left\{v_{r . s . *}\right\} \cap\left\{v_{r . *}\right\}=\emptyset$, and the elements of $\left\{v_{r . *}\right\} \cup\left\{v_{r . s . *}\right\}$ are pairwise distinct. Let $P_{t . s}$ be a set of pattern specifications.

The objects $p_{t},\left(v_{r . *}\right)$, and $\left(v_{r . s . *}\right)$ are called a pattern, element variable specification, and sequence variable specification in $\llbracket p_{t . s} \rrbracket$. The elements of $v_{r . *}$ and $v_{r . s . *}$ are called element pattern variables and sequence pattern variables in $\llbracket p_{t . s} \rrbracket$, respectively.

An element $i_{n s t}$ is an instance in $\llbracket p_{t . s}, s_{b} \rrbracket$ if $\left[\right.$ support $\left.s_{b}\right]=\left\{v_{r . *}\right\},\left[s_{b} v_{r}\right] \in E_{l}$ for $v_{r} \in$ $\left\{v_{r . *}\right\} \backslash\left\{v_{r . s . *}\right\},\left[s_{b} v_{r}\right] \in E_{l . *}$ for $v_{r} \in\left\{v_{r . s . *}\right\}$, and $i_{n s t}$ is an instance in $\llbracket p_{t}, s_{b} \rrbracket$. An element $i_{n s t}$ is an instance in $\llbracket p_{t . s} \rrbracket$ if there exists $s_{b}$ such that $i_{n s t}$ is an instance in $\llbracket p_{t . s}, s_{b} \rrbracket$.

A function $m_{t} \in E_{l} \times P_{t . s} \rightarrow S_{b}$ is a match if the following property holds:

- if $\left[m_{t} e_{l} p_{t . s}\right]=s_{b}$, then $e_{l}$ is an instance in $\llbracket p_{t . s}, s_{b} \rrbracket$.

An element $i_{n s t}$ is an instance in $\llbracket p_{t . s}, m_{t}, s_{b} \rrbracket$ if $\left[m_{t} i_{n s t} p_{t . s}\right]=s_{b}$. An element $i_{n s t}$ is an instance in $\llbracket p_{t . s}, m_{t} \rrbracket$ if there exists $s_{b}$ such that $i_{n s t}$ is an instance in $\llbracket p_{t . s}, m_{t}, s_{b} \rrbracket$.

\subsection{The transition relation}

Let $S_{\text {s.c.c }}$ be a set of conceptual configuration systems. Let $C_{n f}$ be a set of conceptual configurations. An element $t_{r n}$ of the form $\left(c_{n f .1}, c_{n f .2}\right)$ is called a transition. Let $T_{r n}$ be a set of transitions. The configurations $c_{n f .1}$ and $c_{n f .2}$ are called input and output configurations in $\llbracket t_{r n} \rrbracket$.

The transition relations of a IQS is modelled by the transition relation $t_{r n . r l t} \in T_{r n} \rightarrow B_{l}$ based on atomic exogenous transition relations, transition rules, atomic endogenous transition relations, the exogenous transition order and the endogenous transition order. The exogenous transition relation of the IQS is modelled by atomic exogenous transition relations and transition rules. The endogenous transition relation of the IQS is modelled by atomic endogenous transition relations.

Transitions from a configuration $c_{n f}$ in $\llbracket t_{r n . r l t} \rrbracket$ are executed by a program in $\llbracket c_{n f} \rrbracket$. An element sequence $p_{r g}$ is a program in $\llbracket c_{n f} \rrbracket$ if $\left[c_{n f}(0:())::\right.$ state $::$ program $]=\left(p_{r g}\right)$. Let 
$P_{r g}$ be a set of programs. Thus, programs in configurations are specified by the conceptual $(0:())::$ state :: program from the substate program of the configurations. A program in $\llbracket c_{n f} \rrbracket$ is empty if $\left[c_{n f}(0:())::\right.$ state $::$ program $]=()$. Atomic exogenous transition relations and transition rules define transitions executed by the first element of the program. Atomic endogenous transition relations define transitions executed in the case of the empty program.

Let $c_{n f .1} \rightarrow c_{n f .2}$ be a shortcut for $\left[\begin{array}{lll}t_{r n . r l t} & c_{n f .1} & c_{n f .2}\end{array}\right]=$ true. Transitions can return values. An element $v_{l}$ is a value in $\llbracket c_{n f} \rrbracket$ if $v_{l}=\left[c_{n f}(0:())::\right.$ state $::$ value $]$. An element $v_{l}$ is a value in $\llbracket t_{r n} \rrbracket$ if $c_{n f .1} \llbracket t_{r n} \rrbracket \rightarrow c_{n f .2} \llbracket t_{r n} \rrbracket$, and $v_{l}$ is a value in $\llbracket c_{n f .2} \llbracket t_{r n} \rrbracket \rrbracket$. Thus, the returned values in transitions are specified by the conceptual $(0:())::$ state :: value from the substate value of output configurations of the transitions. A transition $t_{r n}$ returns a value $v_{l}$ if $v_{l}$ is a value in $\llbracket t_{r n} \rrbracket$. A transition $t_{r n}$ returns (or generates) an exception $e_{x c}$ if $e_{x c}$ is a value in $\llbracket t_{r n} \rrbracket$. A transition $t_{r n}$ is normally executed if $t_{r n}$ returns no exception.

The special variables conf :: in and val :: in reference to the current configuration and the value in the current configuration, respectively, in the definitions below.

An object $t_{r n . r l t . e x}$ of the form $\left(p_{t},\left(v_{r . *}\right),\left(v_{r . s . *}\right), f_{n}\right)$ is an atomic exogenous transition relation if $\left(p_{t},\left(v_{r . *}\right),\left(v_{r . s . *}\right)\right)$ is a pattern specification, conf :: in $\notin\left\{v_{r . *}\right\} \cup\left\{v_{r . s . *}\right\}$, val :: in $\notin\left\{v_{r . *}\right\} \cup$ $\left\{v_{r . s . *}\right\}, f_{n} \in S_{b} \rightarrow\left(T_{r n} \rightarrow B_{l}\right),\left[\right.$ support $\left.f_{n}\right]=\left\{s_{b}:\left[\right.\right.$ support $\left.s_{b}\right]=\left\{v_{r . *}\right\} \cup\left\{v_{r . s . *}\right\} \cup\{$ conf :: in, val : in $\},\left[s_{b} v_{r}\right] \in E_{l}$ for $v_{r} \in\left\{v_{r . *}\right\}$ and $\left[s_{b} v_{r}\right] \in E_{l . *}$ for $\left.v_{r} \in\left\{v_{r . s . *}\right\}\right\}$. Let $T_{\text {rn.rlt.ex }}$ be a set of atomic exogenous transition relations. Let $c_{n f .1} \rightarrow_{f_{n}, s_{b}} c_{n f .2}$ be a shortcut for $\left.\left[\begin{array}{llll}f_{n} & s_{b}\end{array}\right] c_{n f .1} c_{n f .2}\right]=$ true.

The objects $p_{t},\left(v_{r . *}\right),\left(v_{r . s . *}\right)$, and $f_{n}$ are called a pattern, element variable specification, sequence variable specification, and value in $\llbracket t_{r n . r l t . e x} \rrbracket$. The elements of $v_{r . *}$ and $v_{r . s . *}$ are called element pattern variables and sequence pattern variables in $\llbracket t_{r n . r l t . e x} \rrbracket$, respectively.

A function $t_{r n . r l t . e x . s} \in E_{l} \rightarrow T_{\text {rn.rlt.ex }}$ is called an atomic exogenous transition specification if $\left[\right.$ support $\left.t_{r n . r l t . e x . s}\right]$ is finite. A relation $t_{r n . r l t . e x}$ is an atomic exogenous transition relation in $\llbracket t_{r n . r l t . e x . s} \rrbracket$ if $\left[t_{r n . r l t . e x . s} n_{m}\right]=t_{r n . r l t . e x}$ for some $n_{m} \in E_{l}$. An element $n_{m}$ is a name in $\llbracket t_{r n . r l t . e x}, t_{r n . r l t . e x . s} \rrbracket$ if $\left[t_{r n . r l t . e x . s} n_{m}\right]=t_{r n . r l t . e x}$. An element $n_{m}$ a name in $\llbracket t_{r n . r l t . e x . s} \rrbracket$ if $n_{m}$ is a name in $\llbracket t_{r n . r l t . e x}, t_{r n . r l t . e x . s} \rrbracket$ for some $t_{r n . r l t . e x}$. Let $c_{n f .1} \rightarrow_{n_{m}, s_{b}} c_{n f .2}$ be a shortcut for $c_{n f .1} \rightarrow_{f_{n} \llbracket\left[t_{r n . r l t . e x . s} n_{m}\right] \rrbracket, s_{b}} c_{n f .2}$.

An element $r_{l}$ of the form $\left(p_{t},\left(v_{r . *}\right),\left(v_{r . s . *}\right),\left(b_{d}\right)\right)$ is a transition rule if $b_{d} \in E_{l . *},\left(p_{t},\left(v_{r . *}\right)\right.$, $\left.\left(v_{r . s . *}\right)\right)$ is a pattern specification, conf :: in $\notin\left\{v_{r . *}\right\} \cup\left\{v_{r . s . *}\right\}$, and val :: in $\notin\left\{v_{r . *}\right\} \cup\left\{v_{r . s . *}\right\}$. Let $R_{l}$ be a set of transition rules. 
The objects $p_{t},\left(v_{r . *}\right),\left(v_{r . s . *}\right)$ and $b_{d}$ are called a pattern, element variable specification, sequence variable specification and body in $\llbracket r_{l} \rrbracket$. The elements of $v_{r . *}$ and $v_{r . s . *}$ are called element pattern variables and sequence pattern variables in $\llbracket r_{l} \rrbracket$, respectively.

An attribute element $r_{l . s}$ is called a transition rule specification if $\left[\right.$ support $\left.r_{l . s}\right] \subseteq E_{l}$, and [image $\left.r_{l . s}\right] \subseteq E_{l}$. A rule $r_{l}$ is a rule in $\llbracket r_{l . s} \rrbracket$ if $\left[r_{l . s} n_{m}\right]=r_{l}$ for some $n_{m} \in E_{l}$. An element $n_{m}$ is a name in $\llbracket r_{l}, r_{l . s} \rrbracket$ if $\left[r_{l . s} n_{m}\right]=r_{l}$. An element $n_{m}$ a name in $\llbracket r_{l . s} \rrbracket$ if $n_{m}$ is a name in $\llbracket r_{l}, r_{l . s} \rrbracket$ for some $r_{l}$.

A function $t_{r n . r l t . e n} \in\left\{c_{n f}:\left[c_{n f}(0:())::\right.\right.$ state $::$ program $\left.]=()\right\} \times C_{n f} \rightarrow B_{l}$ is called an atomic endogenous transition relation. Let $T_{r n . r l t . e n}$ be a set of atomic endogenous transition relations.

A function $t_{r n . r l t . e n . s} \in E_{l} \rightarrow T_{r n . r l t . e n}$ is called an atomic endogenous transition specification if [support $t_{r n . r l t . e n . s}$ ] is finite. A relation $t_{r n . r l t . e n}$ is an atomic endogenous transition relation in $\llbracket t_{r n . r l t . e n . s} \rrbracket$ if $\left[t_{r n . r l t . e n . s} n_{m}\right]=t_{r n . r l t . e n}$ for some $n_{m} \in E_{l}$. An element $n_{m}$ is a name in $\llbracket t_{r n . r l t . e n}, t_{r n . r l t . e n . s} \rrbracket$ if $\left[t_{r n . r l t . e n . s} n_{m}\right]=t_{r n . r l t . e n}$. An element $n_{m}$ a name in $\llbracket t_{r n . r l t . e n . s} \rrbracket$

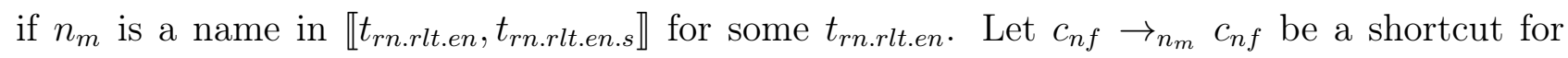
$\left[\left[t_{\text {rn.rlt.en.s }} n_{m}\right] c_{n f} c_{n f .1}\right]=$ true.

Let $\left[\right.$ support $\left.t_{r n . r l t . e x . s}\right],\left[\right.$ support $\left.t_{r n . r l t . e n . s}\right]$ and $\left[\right.$ support $\left.r_{l . s}\right]$ be pairwise disjoint.

An element $o_{r d . t r n . e x}$ of the form $\left(n_{m . *}\right)$ is called an exogenous transition order in $\llbracket t_{r n . r l t . e x . s}$, $r_{l . s} \rrbracket$ if $\left\{n_{m . *}\right\} \subseteq\left[\right.$ support $\left.t_{r n . r l t . e x . s}\right] \cup\left[\right.$ support $\left.r_{l . s}\right]$, and the elements of $n_{m . *}$ are pairwise distinct. It specifies the order of application of atomic exogenous transition relations and transition rules.

An element $o_{r d . t r n . e n}$ of the form $\left(n_{m . *}\right)$ is called an endogenous transition order in $\llbracket t_{r n . r l t . e n . s} \rrbracket$ if $\left\{n_{m . *}\right\} \subseteq$ [support $\left.t_{r n . r l t . e n . s}\right]$, and the elements of $n_{m . *}$ are pairwise distinct. It specifies the order of application of atomic endogenous transition relations.

The information about the transition rule specification and the transition orders is stored in the substate transition of the configurations. The conceptuals (0 : rules) :: state :: transition, $(-1$ : exogenous, 0 : order $)::$ state :: transition and $(-1$ : endogenous, 0 : order $)::$ state :: transition define the transition rule specification, exogenous transition order and endogenous transition order. The conceptual $(0:$ history $)::$ state :: transition defines the substates that store the information about transitions preceding the transition to the current configuration.

An element $c_{n f}$ is consistent with $\left(t_{r n . r l t . e x . s}, r_{l . s}, t_{r n . r l t . e n . s}, o_{r d . t r n . e x}, o_{r d . t r n . e n}\right)$ if the following properties hold:

- if [support $\left.t_{r n . r l t . e x . s}\right] \cap\left[\right.$ support $\left[c_{n f}(0:\right.$ rules $)::$ state $::$ transition $\left.]\right]=\emptyset$; 
- if $\left[\right.$ support $\left.o_{\text {rd.trn.en }}\right] \cap\left[\right.$ support $\left[c_{n f}(0:\right.$ rules $)::$ state $::$ transition $\left.]\right]=\emptyset$;

- if $r_{l . s} \subseteq\left[c_{n f}(0:\right.$ rules $)::$ state :: transition $]$;

- if $n_{m .1} \prec_{\llbracket o_{r d . t r n . e x} \rrbracket} n_{m .2}$, and $n_{m .1}, n_{m .2} \in\left[c_{n f}(-1:\right.$ exogenous, 0 : order $)::$ state ::

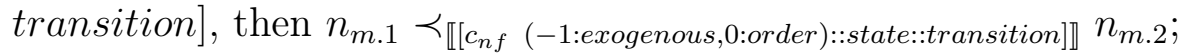

- if $n_{m .1} \prec_{\llbracket o_{r d . t r n . e n} \rrbracket} n_{m .2}$, and $n_{m .1}, n_{m .2} \in\left[c_{n f}(-1:\right.$ endogenous, 0 : order $)::$ state :: transition $]$, then $n_{m .1} \prec_{\llbracket\left[c_{n f}(-1: \text { endogenous,0:order }):: \text { state::transition }\right] \rrbracket} n_{m .2}$.

Let $e_{l . *} \# c_{n f}$ be a shortcut for $\left[c_{n f}\right.$ program.(0:()) : $\left.\left(e_{l . *}\right)\right]$. Let $e_{l . *} \# v_{l} \# c_{n f}$ be a shortcut for $\left[c_{n f}\right.$ program.(0:()) : $\left(e_{l . *}\right)$, value. $\left.(0:()): v_{l}\right]$.

Let $\left[\right.$ add-history $c_{n f .1}$ to $\left.c_{n f .2}\right]$ denote $\left[\right.$ narrow $c_{n f .1}\left[\right.$ support $\left.c_{n f .1}\right] \backslash\left\{\left[c_{n f .1}(0\right.\right.$ : history $)::$ state :: transition $]\}] \cup\left[\right.$ narrow $c_{n f .1}\left\{\left[c_{n f .1}(0\right.\right.$ : history $)::$ state $::$ transition $\left.\left.]\right\}\right]$. A function $t_{r n . r l t} \in C_{n f . c} \times C_{n f} \rightarrow B_{l}$ is a transition relation in $\llbracket t_{r n . r l t . e x . s}, r_{l . s}, t_{r n . r l t . e n . s}, o_{r d . t r n . e x}, o_{r d . t r n . e n} \rrbracket$ if it is defined by the following definition rules (the first proper rule is applied):

- if $c_{n f}$ is not consistent with $\left(t_{r n . r l t . e x . s}, r_{l . s}, t_{r n . r l t . e n . s}, o_{r d . t r n . e x}, o_{r d . t r n . e n}\right)$, then $\left[t_{r n . r l t} c_{n f}\right.$ $\left.c_{n f .1}\right]=$ false;

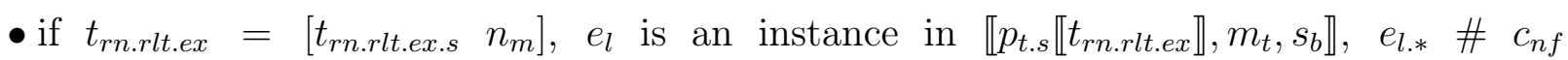
$\rightarrow_{n_{m}, s_{b} \cup\left(\operatorname{conf}:: i n: c_{n f}, v a l:: i n: v_{l} \llbracket c_{n f} \rrbracket\right)} e_{l . * 1} \quad \# \quad v_{l} \# c_{n f .1}$, and $v_{l} \neq$ und, then (executeexogenous-transition, $\left.e_{l},\left(n_{m} n_{m . *}\right)\right), e_{l . *} \# c_{n f} \rightarrow e_{l . * 1} \# v_{l} \# c_{n f .1}$;

- if $t_{r n . r l t . e x}=\left[\begin{array}{lll}t_{r n . r l t . e x . s} & n_{m}\end{array}\right], e_{l}$ is an instance in $\llbracket p_{t . s} \llbracket t_{r n . r l t . e x} \rrbracket, m_{t}, s_{b} \rrbracket, \quad e_{l . *} \# c_{n f}$ $\rightarrow_{n_{m}, s_{b} \cup\left(\operatorname{conf}:: i n: c_{n f}, v a l:: i n: v_{l} \llbracket c_{n f} \rrbracket\right)} e_{l . * .1} \# u_{n d} \# c_{n f .1}$, then (execute-exogenous-transition, $\left.e_{l},\left(n_{m} n_{m . *}\right)\right), e_{l . *} \# c_{n f} \rightarrow\left(\right.$ execute-exogenous-transition, $\left.e_{l},\left(n_{m . *}\right)\right), e_{l . *} \#[$ addhistory $c_{n f .1}$ to $\left.c_{n f}\right]$;

- if $t_{r n . r l t . e x}=\left[t_{r n . r l t . e x . s} n_{m}\right]$, and $e_{l}$ is not an instance in $\llbracket p_{t . s} \llbracket t_{r n . r l t . e x} \rrbracket, m_{t} \rrbracket$, then (executeexogenous-transition, $\left.e_{l},\left(n_{m}, n_{m . *}\right)\right), e_{l . *} \# c_{n f} \rightarrow$ (execute-exogenous-transition, $\left.e_{l},\left(n_{m . *}\right)\right), e_{l . *} \# c_{n f} ;$

- if $r_{l}=\left[\left[c_{n f}(0:\right.\right.$ rules $)::$ state :: transition $\left.] n_{m}\right]$, and $e_{l}$ is an instance in $\llbracket p_{t . s} \llbracket r_{l} \rrbracket, m_{t}, s_{b} \rrbracket$, then (execute-exogenous-transition, $\left.e_{l},\left(n_{m} n_{m . *}\right)\right), e_{l . *} \# c_{n f} \rightarrow\left(\left[\right.\right.$ subst $s_{b} \cup($ conf $::$ in : $c_{n f}$, val :: in : $\left.\left.v_{l} \llbracket c_{n f} \rrbracket\right) b_{d} \llbracket r_{l} \rrbracket\right]$, (execute-exogenous-transition, $e_{l},\left(n_{m} n_{m . *}\right),\left(e_{l . *}\right)$, $\left.c_{n f}\right), e_{l * *} \# c_{n f}$;

- if $v_{l} \neq$ und, then (execute-exogenous-transition, $\left.e_{l},\left(n_{m} n_{m . *}\right),\left(e_{l . * .1}\right), c_{n f .1}\right), e_{l . *} \# v_{l}$ $\# c_{n f} \rightarrow e_{l . *} \# v_{l} \# c_{n f}$;

- (execute-exogenous-transition, $\left.\left(n_{m} n_{m . *}\right), e_{l},\left(e_{l . * .1}\right), c_{n f .1}\right), e_{l . *} \#$ und \# $c_{n f} \rightarrow$ (execute-exogenous-transition, $\left.e_{l},\left(n_{m . *}\right)\right), e_{l . * 1} \#\left[\right.$ add-history $c_{n f}$ to $\left.c_{n f .1}\right]$; 
- if $r_{l}=\left[\left[c_{n f}(0:\right.\right.$ rules $)::$ state :: transition $\left.] n_{m}\right]$, and $e_{l}$ is not an instance in $\llbracket p_{t . s} \llbracket r_{l} \rrbracket, m_{t} \rrbracket$, then (execute-exogenous-transition, $\left.e_{l}, \quad\left(\begin{array}{lll}n_{m} & n_{m . *}\end{array}\right)\right), \quad e_{l . *} \quad \# \quad c_{n f} \rightarrow$ (executeexogenous-transition, $\left.e_{l},\left(n_{m . *}\right)\right), e_{l . *} \# c_{n f}$;

- (execute-exogenous-transition, $\left.e_{l},()\right), e_{l . *} \# c_{n f} \rightarrow e_{l . *} \#$ und $\# c_{n f}$;

- if $t_{r n . r l t . e n}=\left[t_{r n . r l t . e n . s} n_{m}\right], c_{n f} \rightarrow_{n_{m}} e_{l . *} \# v_{l} \# c_{n f .1}$, and $v_{l} \neq u n d$, then (executeendogenous-transition, $\left.\left(n_{m} n_{m . *}\right)\right) \# c_{n f} \rightarrow e_{l . *} \# v_{l} \# c_{n f .1}$;

- if $t_{r n . r l t . e n}=\left[\begin{array}{lll}t_{r n . r l t . e n . s} & n_{m}\end{array}\right]$, and $c_{n f} \rightarrow_{n_{m}} e_{l} e_{l . *} \# u_{n d} \# c_{n f .1}$, then (executeendogenous-transition, $\left.\left(n_{m} n_{m . *}\right)\right) \# c_{n f} \rightarrow e_{l} e_{l . *} \# u_{n d} \# c_{n f .1}$;

- if $t_{\text {rn.rlt.en }}=\left[t_{r n . r l t . e n . s} n_{m}\right]$, and $c_{n f} \rightarrow_{n_{m}} \# u_{n d} \# c_{n f .1}$, then (execute-endogenoustransition, $\left.\left(n_{m} n_{m . *}\right)\right) \# c_{n f} \rightarrow$ (execute-endogenous-transition, $\left.\left(n_{m . *}\right)\right) \#[$ addhistory $c_{n f .1}$ to $\left.c_{n f}\right]$;

- $e_{l}, e_{l . *} \# c_{n f} \rightarrow$ (execute-exogenous-transition, $e_{l},\left[c_{n f}(-1:\right.$ exogenous, 0 : order $)::$ state :: transition]), $e_{l . *} \# c_{n f}$;

- \# $c_{n f} \rightarrow$ (execute-endogenous-transition, $\left[c_{n f}(-1:\right.$ endogenous, 0 : order $)$ :: state :: transition]), \# $c_{n f}$.

\subsection{Conceptual transition systems}

An object $s_{\text {s.t.c }}$ of the form $\left(s_{\text {s.c.c }}, t_{r n . r l t . e x . s}, r_{l . s}, t_{r n . r l t . e n . s}, o_{r d . t r n . e x}, o_{r d . t r n . e n}\right)$ is a conceptual transition system if $s_{\text {s.c.c }}$ is a conceptual configuration system, $t_{r n . r l t . e x . s}, r_{l . s}, t_{r n . r l t . e n . s}, o_{r d . t r n . e x}$ and $o_{\text {rd.trn.en }}$ are an atomic exogenous transition specification, transition rule specification, atomic endogenous transition specification, exogenous transition order and endogenous transition order in $\llbracket A_{t m} \llbracket s_{s . c . c} \rrbracket \rrbracket$, and the sets [support $\left.t_{\text {rn.rlt.ex.s }}\right]$, [support $\left.t_{r n . r l t . e n . s}\right]$ and $\left[\right.$ support $\left.r_{l . s}\right]$ are pairwise disjoint. It specifies the transition system $\left(C_{n f} \llbracket s_{s . c . c} \rrbracket, t_{r n . r l t} \llbracket t_{r n . r l t . e x . s}, r_{l . s}, t_{r n . r l t . e n . s}\right.$, $\left.o_{r d . t r n . e x}, o_{r d . t r n . e n}, m_{t} \llbracket s_{\text {s.c.c }} \rrbracket \rrbracket\right)$. Let $S_{\text {s.t.c }}$ be a set of conceptual transition systems.

The elements of $A_{t m} \llbracket s_{s . c . c} \rrbracket, E_{l} \llbracket s_{s . c . c} \rrbracket, C_{n c p l} \llbracket s_{s . c . c} \rrbracket, S_{t t} \llbracket s_{s . c . c} \rrbracket, C_{n f} \llbracket s_{\text {s.c.c }} \rrbracket$ and $T_{r n} \llbracket A_{t m} \llbracket s_{\text {s.c.c }} \rrbracket \rrbracket$ are called atoms, elements, conceptuals, states, configurations and transitions in $\llbracket s_{\text {s.t.c }} \rrbracket$.

The objects $t_{r n . r l t . e x . s}, r_{l . s}, t_{r n . r l t . e n . s}, o_{r d . t r n . e x}, o_{r d . t r n . e n}, i_{n t r . a . s} \llbracket s_{s . c . c} \rrbracket, d_{f . s} \llbracket s_{s . c . c} \rrbracket, o_{r d . i n t r} \llbracket s_{s . c . c} \rrbracket$ and $m_{t} \llbracket s_{s . c . c} \rrbracket$ are called an atomic exogenous transition specification, transition rule specification, atomic endogenous transition specification, exogenous transition order, endogenous transition order, atomic element interpretation specification, element definition specification, element intepretation order and match in $\llbracket s_{\text {s.t.c }} \rrbracket$.

The function $t_{r n . r l t} \llbracket t_{r n . r l t . e x . s}, r_{l . s}, t_{r n . r l t . e n . s}, o_{r d . t r n . e x}, o_{r d . t r n . e n}, m_{t} \rrbracket$ is called a transition rela- 
tion in $\llbracket s_{s . t . c} \rrbracket$. A system $s_{s . t . c}$ executes $t_{r n}$ if $s_{t t .1} \llbracket t_{r n} \rrbracket \rightarrow s_{t t .2} \llbracket t_{r n} \rrbracket$. A system $s_{s . t . c}$ transits from $s_{t t .1}$ to $s_{t t .2}$ if $s_{s . t . c}$ executes $\left(s_{t t .1}, s_{t t .2}\right)$.

An element $e_{l}$ is interpretable in $\llbracket s_{\text {s.t.c }} \rrbracket$ if $e_{l}$ is interpretable in $\llbracket s_{\text {s.c.c }} \llbracket s_{\text {s.t.c }} \rrbracket \rrbracket$.

An element $e_{l}$ is executable in $\llbracket s_{s . t . c} \rrbracket$ if there exist $n_{m}$ such that $e_{l}$ is an instance in $\llbracket p_{t . s} \llbracket\left[t_{r n . r l t . e x . s} n_{m}\right] \rrbracket, m_{t} \rrbracket$, or $e_{l}$ is an instance in $\llbracket p_{t . s} \llbracket\left[r_{l . s} n_{m}\right] \rrbracket, m_{t} \rrbracket$.

\subsection{Conceptual information transition models}

An object $m_{\text {dl.t.q.c }}$ of the form $\left(s_{\text {s.t.c }}, r_{\text {pr.s. }}, r_{\text {pr.q. }}, r_{\text {pr.a }}\right)$ is a conceptual information transition model in $\llbracket s_{s . t . i} \rrbracket$ if $\left(s_{s . c . c} \llbracket s_{\text {s.t.c }} \rrbracket, r_{\text {pr.s }}, r_{\text {pr.q }}, r_{\text {pr.a }}\right)$ is a conceptual query model in $\llbracket s_{s . q} \llbracket s_{s . t . i} \rrbracket \rrbracket$, $\left[t_{r n . r l t . e x} \llbracket s_{s . t . i} \rrbracket q_{r} a_{n s} s_{t t .1} s_{t t .2}\right]=\left[t_{r n . r l t} \llbracket s_{s . t . c} \rrbracket\left[\left[\begin{array}{ll}r_{\text {pr.s }} & s_{t t .1}\end{array}\right](0:())::\right.\right.$ state $:$ program $\left.:\left(\left[r_{\text {pr.q }} q_{r}\right]\right)\right]$ $\left[\left[\begin{array}{ll}r_{\text {pr.s }} & s_{t t .2}\end{array}\right](0:())::\right.$ state $::$ value $\left.\left.:\left[\begin{array}{ll}r_{\text {pr.a }} & a_{n s}\end{array}\right]\right]\right]$, and $\left[t_{r n . r l t . e n} \llbracket s_{s . t . i} \rrbracket a_{n s} s_{t t .1} s_{t t .2}\right]=\left[t_{r n . r l t} \llbracket s_{s . t . c} \rrbracket\right.$ $\left[\left[r_{\text {pr.s }} s_{t t .1}\right](0:())::\right.$ state :: program : ()] $\left[\left[r_{\text {pr.s }} s_{t t .2}\right](0:())::\right.$ state $::$ value : $\left.\left.\left[r_{p r . a} a_{n s}\right]\right]\right]$. Let $M_{d l . t . q . c}$ be a set of conceptual query transition models.

The objects $s_{\text {s.c.c }} \llbracket s_{\text {s.t.c }} \rrbracket$ and $s_{\text {s.t.c }}$ are called a conceptual configuration system and conceptual transition system in $\llbracket m_{d l . t . q . c} \rrbracket$, respectively. The functions $r_{p r . s}, r_{p r . q}$ and $r_{p r . a}$ are called a state representation, query representation and answer representation in $\llbracket m_{\text {dl.t.q.c. }} \rrbracket$.

A system $s_{\text {s.t.i }}$ is conceptually modelled in $\llbracket s_{\text {s.t.c }} \rrbracket$ if there exists $m_{\text {dl.t.q.c }}$ such that $s_{\text {s.t.c }}=$ $s_{\text {s.t.c }} \llbracket m_{\text {dl.t.q.c. }} \rrbracket$, and $m_{\text {dl.t.q.c }}$ is a conceptual query model in $\llbracket s_{s . t . i} \rrbracket$. The set $\left[\right.$ image $\left.r_{p r . s}\right]$ is called an ontology in $\llbracket s_{\text {s.t.i }}, m_{\text {dl.t.q.c }} \rrbracket$.

\subsection{Extensions}

A system $s_{\text {s.t.i.1 }}$ is an extension of $s_{\text {s.t.i. } 2}$ if $s_{\text {s.q }} \llbracket s_{\text {s.t.i.1 }} \rrbracket$ is an extension of $s_{\text {s.q }} \llbracket s_{\text {s.t.i.2 }} \rrbracket$, and $s_{t} \llbracket s_{\text {s.t.i.1 }} \rrbracket \subseteq s_{t} \llbracket s_{\text {s.t.i.2 }} \rrbracket$ for each $s_{t} \in\left\{t_{\text {rn.rlt.ex }}, t_{\text {rn.rlt.en }}\right\}$.

A system $s_{\text {s.t.c. } 1}$ is an extension of $s_{\text {s.t.c. } 2}$ if $s_{\text {s.c.c }} \llbracket s_{\text {s.t.c. } 1} \rrbracket$ is an extension of $s_{\text {s.c.c }} \llbracket s_{\text {s.t.c. } 2} \rrbracket$, $s_{t} \llbracket s_{s . t . c .1} \rrbracket \subseteq s_{t} \llbracket s_{\text {s.t.c. } 2} \rrbracket$ for each $s_{t} \in\left\{t_{r n . r l t . e x . s}, r_{l . s}, t_{r n . r l t . e n . s}\right\}$, and the following property hold:

- if $\quad n_{m .1} \prec \llbracket o_{r d . t r n . e x} \llbracket s_{s . t . c .1} \rrbracket \quad n_{m .2}, \quad$ and $n_{m .1}, \quad n_{m .2} \in \quad o_{r d . t r n . e x} \llbracket s_{s . t . c .2} \rrbracket$, then $n_{m .1} \prec \llbracket o_{\text {rd.trn.ex }} \llbracket s_{\text {s.t.c. } 2 \rrbracket} n_{m .2} ;$

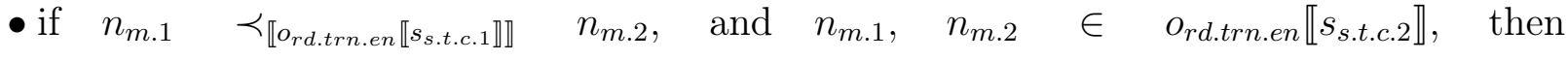
$n_{m .1} \prec \llbracket o_{\text {rd.trn.en }} \llbracket s_{\text {s.t.c. } 2 \rrbracket \rrbracket} n_{m .2}$.

A CCS $l_{n}$ is a language of CTSs if the conceptual structures (atoms, elements, conceptuals and so on) of $l_{n}$ is syntactically defined.

\subsection{Programs}


A program $p_{r g}$ is executed in $\llbracket t_{r n} \rrbracket$ if $p_{r g}$ is a program in $\llbracket c_{n f .1} \llbracket t_{r n} \rrbracket \rrbracket$, and $c_{n f .1} \llbracket t_{r n} \rrbracket \rightarrow$ $c_{n f .2} \llbracket t_{r n} \rrbracket$. A program $p_{r g}$ executes (initiates) $t_{r n}$ if $p_{r g}$ is executed in $\llbracket t_{r n} \rrbracket$.

An element $v_{l}$ is a value in $\llbracket p_{r g}, t_{r n} \rrbracket$ if $p_{r g}$ executes $\llbracket t_{r n} \rrbracket$, and $v_{l}$ is a value in $\llbracket t_{r n} \rrbracket$. A program $p_{r g}$ returns $v_{l}$ in $\llbracket t_{r n} \rrbracket$ if $v_{l}$ is a value in $\llbracket p_{r g}, t_{r n} \rrbracket$. A program $p_{r g}$ returns $v_{l}$ in $\llbracket c_{n f} \rrbracket$ if there exists $t_{r n}$ such that $p_{r g}$ returns $v_{l}$ in $\llbracket t_{r n} \rrbracket$, and $c_{n f}=c_{n f .1} \llbracket t_{r n} \rrbracket$.

A program $p_{r g}$ returns (or generates) an exception $e_{x c}$ in $\llbracket t_{r n} \rrbracket$ if $e_{x c}$ is a value in $\llbracket p_{r g}, t_{r n} \rrbracket$. A program $p_{r g}$ is normally executed in $\llbracket t_{r n} \rrbracket$ if $p_{r g}$ is executed in $\llbracket t_{r n} \rrbracket$, and $t_{r n}$ is normally executed.

An element $e_{l}$ is executed in $\llbracket t_{r n} \rrbracket$ if there exist $p_{r g}$ such that $p_{r g}$ is executed in $\llbracket t_{r n} \rrbracket$, and $e_{l}=\left[p_{r g} \cdot 1\right]$. An element $e_{l}$ executes (initiates) $t_{r n}$ if $e_{l}$ is executed in $\llbracket t_{r n} \rrbracket$.

An element $v_{l}$ is a value in $\llbracket e_{l}, t_{r n} \rrbracket$ if $e_{l}$ is executed in $\llbracket t_{r n} \rrbracket$, and $v_{l}$ is a value in $\llbracket t_{r n} \rrbracket$. An element $v_{l}$ returns $v_{l}$ in $\llbracket t_{r n} \rrbracket$ if $v_{l}$ is a value in $\llbracket v_{l}, t_{r n} \rrbracket$. An element $v_{l}$ returns $v_{l}$ in $\llbracket c_{n f} \rrbracket$ if there exists $t_{r n}$ such that $v_{l}$ returns $v_{l}$ in $\llbracket t_{r n} \rrbracket$, and $c_{n f}=c_{n f .1} \llbracket t_{r n} \rrbracket$.

An element $e_{l}$ returns (or generates) an exception $e_{x c}$ in $\llbracket t_{r n} \rrbracket$ if $e_{x c}$ is a value in $\llbracket e_{l}, t_{r n} \rrbracket$. An element $e_{l}$ is normally executed in $\llbracket t_{r n} \rrbracket$ if $e_{l}$ is executed in $\llbracket t_{r n} \rrbracket$, and $t_{r n}$ is normally executed.

\subsection{Safe configurations, transitions, programs and elements}

A configuration $c_{n f}$ is locally safe if $v_{l} \llbracket c_{n f} \rrbracket \neq u n d$.

A transition $t_{r n}$ is safe if $c_{n f .1} \llbracket t_{r n} \rrbracket$ and $c_{n f .2} \llbracket t_{r n} \rrbracket$ are locally safe.

A configuration $c_{n f}$ is safe if there is no $c_{n f .1}$ such that $c_{n f} \rightarrow^{*} c_{n f .1}$ and $c_{n f .1}$ is not locally safe.

A program $p_{r g}$ is safe in $\llbracket c_{n f} \rrbracket$ if $p_{r g}$ is a program in $\llbracket c_{n f} \rrbracket$, and $c_{n f}$ is safe. A program $p_{r g}$ is safe if $p_{r g}$ is safe in $\llbracket c_{n f} \rrbracket$ for each $c_{n f}$.

An element $e_{l}$ is safe in $\llbracket c_{n f} \rrbracket$ if $e_{l}=\left[p_{r g} \llbracket c_{n f} \rrbracket\right.$. 1], and $p_{r g}$ is safe in $\llbracket c_{n f} \rrbracket$. An element $e_{l}$ is safe if $e_{l}$ is safe in $\llbracket c_{n f} \rrbracket$ for each $c_{n f}$.

\section{The CTSL language}

The CTSL language (Conceptual Transition System Language) is a basic language of CTSs. The CCSL language is a sublanguage of CTSL. Interpretable and executable elements of CTSL are called basic elements of CTSs.

Let $s_{b} \subseteq\left(x: x_{0}, y: y_{0}, z: z_{0}, u: u_{0}, v: v_{0}, w: w_{0}, x_{1}: x_{1.0}, \ldots, x_{n_{t}}: x_{n_{t} .0}\right.$, conf :: in : $c_{n f}$, val :: in $\left.: v_{l} \llbracket c_{n f} \rrbracket\right)$. 


\subsection{Syntax of CCSL}

CTSL is an extension of CCSL. Therefore, atoms, elements, conceptual states, conceptual configurations, pattern specifications and element definitions are represented in CTSL as in CCSL.

The element (rule $p_{t} \operatorname{var}\left(v_{r . *}\right)$ seq $\left(v_{r . s . *}\right)$ then $\left.b_{d}\right)::$ name $:: n_{m}$ in CCSL represents the transition rule $\left(p_{t},\left(v_{r . *}\right),\left(v_{r . s . *}\right), b_{d}\right)$ with the name $n_{m}$.

For simplicity, we omit the names of atomic transition relations and transition rules.

\subsection{The special forms for atomic exogenous transition relations, transition rules and atomic endogenous transition relations}

In this section we define the special forms for atomic exogenous transition relations, transition rules and atomic endogenous transition relations used below.

The form (transition $p_{t}$ var $\left(v_{r . *}\right) \operatorname{seq}\left(v_{r . s . *}\right)$ then $\left.f_{n}\right)::$ name $:: n_{m}$ denotes the atomic exogenous transition relation $\left(p_{t},\left(v_{r . *}\right),\left(v_{r . s . *}\right), f_{n}\right)$ with the name $n_{m}$.

The objects $\operatorname{var}\left(v_{r . *}\right)$ and $\operatorname{seq}\left(v_{\text {r.s.* }}\right)$ in the form (transition ...) can be omitted. The omitted objects correspond to var () and seq (), respectively.

The form (endogenous-transition $f_{n}$ ) :: name :: $n_{m}$ denotes the atomic endogenous transition relation $f_{n}$ with the name $n_{m}$.

Let $\left\{v_{r . *}\right\},\left\{v_{r . s . *}\right\},\left\{v_{r . * .1}\right\}$ and $\left\{v_{r . * .2}\right\}$ are pairwise disjoint, $\left\{v_{r . * .3}\right\} \subseteq\left\{v_{r . *}\right\} \cup\left\{v_{r . * .1}\right\} \cup\left\{v_{r . * 2}\right\}$, and $\left(e_{l . *}\right) \in\{()$, und,abn $\}$. The form (rule p $\operatorname{var}\left(v_{r . *}\right)$ seq $\left(v_{r . s . *}\right)$ abn $\left(v_{r . * .1}\right)$ und $\left(v_{r . * .2}\right)$ val $\left(v_{r . * 3}\right) e_{l . *}$ where $c_{n d}$ then $\left.b_{d}\right)$ called a rule form is defined as follows:

- $\left(\right.$ rule $p_{t} \operatorname{var}\left(v_{r . *}\right) \operatorname{seq}\left(v_{r . s . *}\right)$ und $\left(v_{r . * .1}\right)$ abn $\left(v_{r . * .2}\right)$ val $\left(v_{r . * .3}\right) e_{l . *}$ where $c_{n d}$ then $\left.b_{d}\right)$ is a shortcut for (rule p $p_{t} \operatorname{var}\left(v_{r . *}\right) \operatorname{seq}\left(v_{r . s . *}\right)$ abn $\left(v_{r . * .1}\right)$ und $\left(v_{r . * .2}\right)$ val $\left(v_{r . * .3}\right) e_{l . *}$ then $\left(\right.$ if $c_{n d}$ then $b_{d}$ else und));

- $\left(\right.$ rule $p_{t} \operatorname{var}\left(v_{r . *}\right) \operatorname{seq}\left(v_{r . s . *}\right)$ und $\left(v_{r . * .1}\right)$ abn $\left(v_{r . * .2}\right)$ val $\left(v_{r . * .3}, v_{r}\right) e_{l . *}$ then $\left.b_{d}\right)$ is a shortcut for $\left(\right.$ rule $p_{t} \operatorname{var}\left(v_{r . *}\right) \operatorname{seq}\left(v_{r . s . *}\right)$ und $\left(v_{r . * .1}\right)$ abn $\left(v_{r . * .2}\right)$ val $\left(v_{r . * .3}\right) e_{l . *}$ then (let $w$ be $v_{r}$ in [subst $\left.\left.\left.\left(v_{r}:: *: w\right) b_{d}\right]\right)\right)$, where $w$ is a new element that does not occur in this definition;

- $\left(\right.$ rule $p_{t} \operatorname{var}\left(v_{r . *}\right) \operatorname{seq}\left(v_{r . s . *}\right)$ und $\left(v_{r . * 1}\right)$ abn $\left(v_{r . * 2}\right)$ val ()$e_{l . *}$ then $\left.b_{d}\right)$ is a shortcut for (rule $p_{t} \operatorname{var}\left(v_{r . *}\right) \operatorname{seq}\left(v_{r . s . *}\right)$ und $\left(v_{r . * .1}\right)$ abn $\left(v_{r . * 2}\right) e_{l . *}$ then $\left.b_{d}\right)$;

- $\left(\right.$ rule $p_{t} \operatorname{var}\left(v_{r . *}\right) \operatorname{seq}\left(v_{r . s . *}\right)$ und $\left(v_{r . * 1}, v_{r}\right)$ abn $\left(v_{r . * .2}\right) e_{l . *}$ then $\left.b_{d}\right)$ is a shortcut for (rule $p_{t} \operatorname{var}\left(v_{r . *}\right) \operatorname{seq}\left(v_{r . s . *}\right)$ und $\left(v_{r . * .1}\right)$ abn $\left(v_{r . * .2}\right) e_{l . *}$ then (if $\left(v_{r}\right.$ is undefined) then und else $\left.b_{d}\right)$ ); 
- $\left(\right.$ rule $p_{t} \operatorname{var}\left(v_{r . *}\right) \operatorname{seq}\left(v_{r . s . *}\right)$ und () abn $\left(v_{r . * .2}\right) e_{l . *}$ then $\left.b_{d}\right)$ is a shortcut for (rule $p_{t}$ var $\left(v_{r . *}\right) \operatorname{seq}\left(v_{r . s . *}\right)$ abn $\left(v_{r . * .2}\right) e_{l . *}$ then $\left.b_{d}\right)$;

- $\left(\right.$ rule $p_{t} \operatorname{var}\left(v_{r . *}\right) \operatorname{seq}\left(v_{r . s . *}\right)$ abn $\left(v_{r . * 2}, v_{r}\right) e_{l . *}$ then $\left.b_{d}\right)$ is a shortcut for (rule $p_{t} \operatorname{var}\left(v_{r . *}\right)$ $\operatorname{seq}\left(v_{r . s . *}\right)$ abn $\left(v_{r . * .2}\right) e_{l . *}$ then (if $\left(v_{r}\right.$ is abnormal) then $v_{r}$ else $\left.\left.b_{d}\right)\right)$;

- (rule p $p_{t} \operatorname{var}\left(v_{r . *}\right) \operatorname{seq}\left(v_{r . s . *}\right)$ abn ()$e_{l . *}$ then $\left.b_{d}\right)$ is a shortcut for (rule p $p_{t}$ var $\left(v_{r . *}\right)$ seq $\left(v_{r . s . *}\right) e_{l . *}$ then $\left.b_{d}\right)$;

- $\left(\right.$ rule $p_{t} \operatorname{var}\left(v_{r . *}\right) \operatorname{seq}\left(v_{r . s . *}\right)$ und then $\left.b_{d}\right)$ is a shortcut for (rule p $p_{t} \operatorname{var}\left(v_{r . *}\right) \operatorname{seq}\left(v_{r . s . *}\right)$ then (if (val :: in is undefined) then skip else $b_{d}$ );

- $\left(\right.$ rule $p_{t} \operatorname{var}\left(v_{r . *}\right) \operatorname{seq}\left(v_{r . s . *}\right)$ abn then $\left.b_{d}\right)$ is a shortcut for $\left(r u l e p_{t} \operatorname{var}\left(v_{r . *}\right)\right.$ seq $\left(v_{r . s . *}\right)$ then (if (val :: in is abnormal) then skip else $b_{d}$ ).

The element $c_{n d}$ specifies the restriction on the values of the pattern variables. The undefined value is propagated through the variables of $v_{r * * .1}$. Abnormal values are propagated through the variables of $v_{r . * 2}$. The sequence $e_{l . *}$ specifies propagation of abnormal values depending on the value of val :: in. The undefined value is propagated when $e_{l . *}=$ und. Abnormal values are propagated when $e_{l . *}=a b n$. The special element $v_{r}:: *$ references to the value of element associated with the pattern variable $v_{r}$. A pattern variable is evaluated if the element associated with it is evaluated. Thus, the sequence $v_{r . * .3}$ contains evaluated pattern variables. A pattern variable is quoted if the element associated with it is not evaluated. Let $F_{r m . r}$ be a set of rule forms.

The objects $\operatorname{var}\left(v_{r . *}\right)$, seq $\left(v_{r . s . *}\right)$, und $\left(v_{r . * 1}\right)$, abn $\left(v_{r . * .2}\right)$, val $\left(v_{r . * .3}\right)$ and where $c_{n d}$ in the form (rule ...) can be omitted. The omitted objects correspond to var (), seq (), und (), abn (), val () and where true, respectively.

\section{Semantics of executable elements in CTSL}

\subsection{Element interpretation}

The element $x::$ value returning the interpretation of $x$ is defined by the rule (rule $x::$ value var $(x)$ abn then $x::$ value :: atm);

(transition $x::$ value :: atm var $(x)$ then $\left.f_{n}\right)$, where $x_{0}$ :: value :: atm $e_{l . *} \# c_{n f} \rightarrow_{f_{n}, s_{b}} e_{l . *} \#\left[\right.$ value $\left.x_{0} c_{n f}\right] \# c_{n f}$.

\subsection{Abnormal elements operations}

The element und is defined by the rule 
(rule und abn then und :: q).

The element $e_{x c}$ is defined by the rule

(rule $x$ var $(x)$ abn where ( $x$ is exception) then $x:: q)::$ name :: ("@", exception).

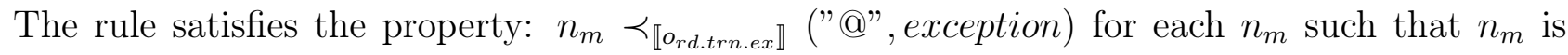
a name of an atomic exogenous transition relation or transition rule with the pattern distinct from $v_{r}$, where $v_{r}$ is a variable of this pattern.

The element $e_{l}:: q$ is defined by the rule (rule $x:: q$ var $(x)$ abn then $x:: q::$ value) .

The element $e_{l}$ of the form (catch :: $u x y$ ) called an undefined value handler is defined as follows:

(transition (catch :: $u$ x $y$ ) var $(x)$ seq $(y)$ then $\left.f_{n}\right)$, where $\left(\operatorname{catch}:: \quad u \quad x_{0} y_{0}\right), e_{l . *} \# v_{l} \# c_{n f} \rightarrow_{f_{n}, s_{b}}\left[\operatorname{subst}\left(x_{0}: v_{l}\right) y_{0}\right], e_{l . *} \#$ true \# $c_{n f}$. The elements $x$ and $y$ are called a variable and body in $\llbracket e_{l} \rrbracket$. The element $e_{l}$ replaces all occurences of $x$ in $y$ by the current value, resets the current value to true and executes the modified body.

The element $e_{l}$ of the form ( $\operatorname{catch} x y$ ) called an exception handler is defined as follows: (rule (catch $x y)$ var $(x)$ seq $(y)$ und then (catch :: $u x y)$ ).

The elements $x$ and $y$ are called a variable and body in $\llbracket e_{l} \rrbracket$.

The element $e_{l}$ of the form (throw $x$ ) is defined by the rule (rule (throw $x)$ var $(x)$ val $(x)$ abn then (throw $x:: *)::$ atm); (transition (throw $x)$ :: atm var $(x)$ then $f_{n}$ ), where $\left(\right.$ throw $\left.x_{0}\right)::$ atm, $e_{l . *} \# c_{n f} \rightarrow_{f_{n}, s_{b}} e_{l . *} \# x_{0} \# c_{n f}$. The element $x$ is called a body in $\llbracket e_{l} \rrbracket$.

The deletion (delete-exception $x$ ) of the exception of the type $x$ is defined by the rule (rule (delete-exception $x$ ) var $(x)$ und then (catch $w$

(if $((w$ is exception $)$ and $((($ element in $w)$.. type $)=x:: q))$

then (throw true) else (throw $w:: q)))$ ).

\subsection{Statements}

The element skip is defined as follows:

(rule skip abn then skip :: atm);

(transition skip :: atm then $f_{n}$ ),

where skip :: atm, $e_{l . *} \# c_{n f} \rightarrow_{f_{n}, s_{b}} e_{l . *} \# c_{n f}$. 
The sequential composition $e_{l}$ of the form $\left(s e q e_{l . *}\right)$ is defined by the rule (rule $(\operatorname{seq} x)$ var $(x) \operatorname{seq}(x)$ then $x)$

The elements of $e_{l . *}$ are called elements in $\llbracket e_{l} \rrbracket$ and $e_{l . *}$ is called a body in $\llbracket e_{l} \rrbracket$. The element $e_{l}$ executes its elements sequentially from left to right.

The conditional element (if $x$ then $y$ else $z$ ) is defined as follows:

(rule (if $x$ then $y$ else $z)$ var $(x) \operatorname{seq}(y, z)$ val $(x)$ abn

then (if $x:: *$ then $y$ else $z)::$ atm);

(transition (if $x$ then $y$ else $z$ ) :: atm var $(x) \operatorname{seq}(y, z)$ then $\left.f_{n}\right)$, where $\left(\right.$ if $x_{0}$ then $y_{0}$ else $\left.z_{0}\right)::$ atm, $e_{l . *} \# c_{n f} \rightarrow_{f_{n}, s_{b}}\left[\right.$ if $\left[x_{0} \neq\right.$ und $]$ then $y_{0}$ else $\left.z_{0}\right], e_{l . *} \# c_{n f}$.

The element (if $x$ then $y$ ) is a shortcut for (if $x$ then $y$ else skip).

The conditional element (if $x$ then $y$ elseif $z$ then $u$... else $v$ ) is defined as follows:

(definition (if $x$ then $y$ elseif $z) \operatorname{var}(x) \operatorname{seq}(y, z)$ abn

then (if $x$ then $y$ else $($ if $z))$ ).

The element $e_{l}$ of the form (let $x$ be $y$ in $z$ ) is defined as follows:

(rule (let $x$ be $y$ in $z)$ var $(x) \operatorname{seq}(y, z)$ abn then (let $x$ be $y$ in $z):: a t m)$;

(transition (let $x$ be $y$ in $z)$ :: atm var $(x) \operatorname{seq}(y, z)$ then $\left.f_{n}\right)$,

where $\left(\right.$ let $x_{0}$ be $y_{0}$ in $\left.z_{0}\right)::$ atm, $e_{l . *} \# c_{n f} \rightarrow_{f_{n}, s_{b}} y_{0}$, (let $x_{0}$ be-val-in $\left.z_{0}\right), e_{l . *} \# c_{n f}$. The elements $x, y$ and $z$ are called a substitution variable, substitution value and substitution body in $\llbracket e_{l} \rrbracket$.

The auxiliary element (let $x$ be-val-in $y$ ) is defined as follows: (transition (let $x$ be-val-in $y$ ) var $(x)$ seq $(y)$ abn then $f_{n}$ ), where $\left(l e t x_{0}\right.$ be-val-in $\left.y_{0}\right), e_{l . *} \# v_{l} \# c_{n f} \rightarrow_{f_{n}, s_{b}}\left[\operatorname{subst}\left(x_{0}: v_{l}\right) y_{0}\right], e_{l . *} \# c_{n f}$.

The element $e_{l}$ of the form (let :: seq $x$ be $y$ in $z$ ), where $x \in E_{l .(*)}, y \in E_{l .(*)}$, and $[$ len $x]=[$ len $y]$, is defined by the rule (rule (let :: seq $x, y$ be $(z), u$ in $v)$ var $(x) \operatorname{seq}(y, z, u, v)$ abn then (let $x$ be $z$ in (let :: seq $y$ be $u$ in $v))$ ); (rule (let:: seq be in $v$ ) seq $(v)$ abn then $v$ ).

The elements $x, y$ and $z$ are called a substitution variables specification, substitution values specification and substitution body in $\llbracket e_{l} \rrbracket$. The elements of $x$ and $y$ are called substitution variables and substitution values in $\llbracket e_{l} \rrbracket$.

The iterator $e_{l}$ of the form (while $x$ do $y$ ) is defined by the rule (if (while $x$ do $y$ ) var $(x)$ seq $(y)$ abn then (if $x$ then $y$ (while $x$ do $y))$ ). 
The elements $x$ and $y$ are called a condition and body in $\llbracket e_{l} \rrbracket$.

The iterator $e_{l}$ of the form (foreach $x$ in $y$ do $z$ ) is defined as follows:

(rule (foreach $x$ in $y$ do $z)$ var $(x, y)$ seq $(z)$ val $(y)$ abn where $(y:: *$ is sequence) then (foreach $1 x$ in $y:: *$ do $z)$ ).

The objects $x, y$ and $z$ are called an iteration variable, iteration structure specifier and body in $\llbracket e_{l} \rrbracket$. The element $e_{l}$ executes sequentially $z$ for values of $x$ from $e_{l .1}$, where $e_{l .1}$ is the value of $y$.

The element (foreach $1 x$ in $y$ do $z$ ) is defined by the rules (rule (foreach $1 x$ in () do y) var $(x)$ seq (y) abn then); (rule (foreach $1 x$ in $(y z)$ do $v)$ var $(x, y) \operatorname{seq}(z, v)$ abn then (let $x$ be $y$ in $v)$, (foreach $1 x$ in $(z)$ do $v)$ ).

\subsection{Characteristic functions for defined concepts}

An object $d_{f . c}$ is a concept definition if $d_{f . c}$ is an atomic transition relation of the form (transition $n_{m}$ if $\left(e_{l .1}\right.$ is $\left.e_{l .2}\right)$ var $\left(v_{r . *}\right)$ seq $\left(v_{r . s . *}\right)$ then $f_{n}$ ), or $d_{f . c}$ is a transition rule of the form (rule $n_{m}$ if ( $e_{l .1}$ is $\left.e_{l .2}\right) \operatorname{var}\left(v_{r . *}\right) \operatorname{seq}\left(v_{r . s . *}\right)$ then $\left.b_{d}\right)$. Concept definitions specify concepts and their instances. Concepts specified by them are called defined concepts. The elements $e_{l .1}$ and $e_{l .2}$ are called an instance pattern and concept pattern in $\llbracket d_{f . c} \rrbracket$. The element $\left(e_{l .1}\right.$ is $\left.e_{l .2}\right)$ is called a characteristic function in $\llbracket d_{f . c} \rrbracket$. Let $D_{f . c}$ be a set of concept definitions.

An element $c_{n c p . d}$ is a defined concept in $\llbracket d_{f . c}, s_{b} \rrbracket$ if $c_{n c p}$ is an instance in $\llbracket\left(e_{l .2}, \operatorname{var}\left(v_{r . *}\right)\right.$ seq $\left.\left(v_{r . s . *}\right)\right), m_{t}, s_{b} \rrbracket$. An element $c_{n c p . d}$ is a defined concept in $\llbracket d_{f . c} \rrbracket$ if there exists $s_{b}$ such that $c_{n c p . d}$ is a concept in $\llbracket d_{f . c}, s_{b} \rrbracket$. An element $c_{n c p . d}$ is a defined concept in $\llbracket c_{n f} \rrbracket$ if there exists $d_{f . c} \llbracket c_{n f} \rrbracket$ such that $c_{n c p . d}$ is a concept in $\llbracket d_{f . c} \rrbracket$. Let $C_{n c p . d}$ be a set of defined concepts.

An element $i_{n s t n}$ is an instance in $\llbracket d_{f . c}, s_{b} \rrbracket$ if $i_{n s t n}$ is an instance in $\llbracket\left(e_{l .1}, \operatorname{var}\left(v_{r . *}\right) \operatorname{seq}\left(v_{r . s . *}\right)\right)$, $m_{t}, s_{b} \rrbracket$. An element $i_{n s t n}$ is an instance in $\llbracket d_{f . c} \rrbracket$ if there exists $s_{b}$ such that $c_{n c p . d}$ is an instance in $\llbracket d_{f . c}, s_{b} \rrbracket$.

An element $i_{n s t n}$ is an instance in $\llbracket c_{n c p . d}, c_{n f}, d_{f . c} \rrbracket$ if $i_{n s t n}$ is an instance in $\llbracket d_{f . c} \rrbracket, c_{n c p . d}$ is a defined concept in $\llbracket d_{f . c} \rrbracket$, and there exist $c_{n f .1}$ and $v_{l}$ such that (execute-exogenous-transition, $\left(i_{n s t n}\right.$ is $\left.\left.c_{n c p . d}\right),\left(n_{m}\right)\right) \# c_{n f} \rightarrow^{*} \# v_{l} \# c_{n f .1}$, and $v_{l} \neq u n d$. An element $i_{n s t n}$ is an instance in $\llbracket c_{n c p . d}, c_{n f} \rrbracket$ if there exists $d_{f . c}$ such that $i_{n s t n}$ is an instance in $\llbracket c_{n c p . d}, c_{n f}, d_{f . c} \rrbracket$. An element $c_{n c p . d}$ is an instance in $\llbracket c_{n f} \rrbracket$ if there exists $c_{n c p . d}$ such that $i_{n s t n}$ is an instance in $\llbracket c_{n c p . d}, c_{n f} \rrbracket$. Let $I_{n s t n}$ be a set of instances. 
A set $s_{t}$ is called a content in $\llbracket c_{n c p . d}, c_{n f} \rrbracket$ if $s_{t}$ is a set of all $i_{n s t n}$ such that $i_{n s t n}$ is an instance in $\llbracket c_{n c p . d}, c_{n f} \rrbracket$. Let $\left[\right.$ content $\left.c_{n c p . d} c_{n f}\right]$ denote the content in $\llbracket c_{n c p . d}, c_{n f} \rrbracket$.

The notion of defined concepts is extended to the rules of the form (rule $\left(e_{l .1}\right.$ is $\left.e_{l .2}\right) \operatorname{var}\left(v_{r . *}\right)$ $\operatorname{seq}\left(v_{r . s . *}\right)$ und $\left(v_{r . * .1}\right)$ val $\left(v_{r . * 3}\right)$ where $c_{n d}$ then $\left.b_{d}\right)$. Let $r_{l}$ have this form. An element $c_{n c p . d}$ is a defined concept in $\llbracket r_{l}, s_{b} \rrbracket$ if $c_{n c p . d}$ is a defined concept in $\llbracket r_{l .1}, s_{b} \rrbracket$, where $r_{l .1}$ is a rule of the form $\left(\right.$ rule $\left(e_{l .1}\right.$ is $\left.e_{l .2}\right) \operatorname{var}\left(v_{r . *}\right) \operatorname{seq}\left(v_{r . s . *}\right)$ then $\left.b_{d .1}\right)$ such that $r_{l}$ is reduced to $r_{l .1}$.

The element ( $x$ is atom) specifying that $x$ is an atom is defined by the rule (rule $(x$ is atom) var $(x)$ abn then $(x$ is atom) :: value).

The element ( $x$ is update) specifying that $x$ is an element update is defined by the rule (rule ( $x$ is update) var $(x)$ abn then ( $x$ is update) :: value).

The element ( $x$ is multi-attribute) specifying that $x$ is a multi-attribute element is defined by the rule

(rule ( $x$ is multi-attribute) var $(x)$ abn then ( $x$ is multi-attribute) :: value).

The element ( $x$ is attribute) specifying that $x$ is an attribute element is defined by the rule (rule ( $x$ is attribute) var $(x)$ abn then $(x$ is attribute) :: value).

The element ( $x$ is sorted) specifying that $x$ is a sorted element is defined by the rule (rule ( $x$ is sorted) var $(x)$ abn then ( $x$ is sorted) :: value).

The element ( $x$ is undefined) specifying that $x$ equals und is defined by the rule (rule ( $x$ is undefined) var $(x)$ abn then ( $x$ is undefined) :: value).

The element ( $x$ is defined) specifying that $x$ does not equal und is defined by the rule (rule ( $x$ is defined) var $(x)$ abn then ( $x$ is defined) :: value).

The element ( $x$ is exception) specifying that $x$ is an exception is defined by the rule (rule ( $x$ is exception) var $(x)$ abn then ( $x$ is exception) :: value).

The element ( $x$ is normal) specifying that $x$ is a normal element is defined by the rule (rule ( $x$ is normal) var $(x)$ abn then $(x$ is normal) :: value.

The element ( $x$ is normal) specifying that $x$ is an abnormal element is defined by the rule (rule ( $x$ is abnormal) var $(x)$ abn then $(x$ is abnormal) :: value.

The element ( $x$ is sequence) specifying that $x$ is a sequence element is defined by the rule (rule ( $x$ is sequence) var $(x)$ abn then ( $x$ is sequence) :: value).

The element ( $x$ is set) specifying that the elements of the sequence element $x$ are pairwise distinct is defined as follows:

(rule ( $x$ is set) var $(x)$ abn then ( $x$ is set) :: value). 
The element ( $x$ is empty) specifying that $x$ is an empty element is defined by the rule (rule ( $x$ is empty) var $(x)$ abn then $(x$ is empty) :: value).

The element ( $x$ is nonempty) specifying that $x$ is not an empty element is defined by the rule

(rule ( $x$ is nonempty) var $(x)$ abn then ( $x$ is nonempty) :: value).

The element ( $x$ is conceptual) specifying that $x$ is a conceptual is defined by the rule (rule ( $x$ is conceptual) var $(x)$ abn then $(x$ is conceptual) :: value).

The element ( $x$ is (conceptual in $y)$ ) specifying that $x$ is a conceptual in the context of the state $y$ is defined by the rule (rule $(x$ is (conceptual in $y))$ var $(x, y)$ abn then $(x$ is (conceptual in $y))$ :: value.

The element ( $x$ is state) specifying that $x$ is a conceptual state is defined by the rule (rule ( $x$ is state) var $(x)$ abn then ( $x$ is state) :: value).

The element ( $x$ is configuration) specifying that $x$ is a conceptual configuration is defined by the rule (rule ( $x$ is configuration) var $(x)$ abn then ( $x$ is configuration) :: value).

The element ( $x$ is nat) specifying that $x$ is a natural number is defined by the rule (rule ( $x$ is nat) var $(x)$ abn then ( $x$ is nat) :: value).

The element ( $x$ is nat 0 ) specifying that $x$ is either a natural number, or a zero is defined by the rule

(rule ( $x$ is nat0) var $(x)$ abn then $(x$ is nat0) :: value).

The element ( $x$ is int) specifying that $x$ is an integer is defined by the rule (rule ( $x$ is int) var $(x)$ abn then ( $x$ is int) :: value).

The element ( $x$ is (satisfiable in $y)$ ) specifying that $x$ is satisfiable in the context of variables $y$ is defined by the rule

(rule $(x$ is (satisfiable in $y)$ ) var $(x)$ seq $(y)$ abn

then ( $x$ is (satisfiable in $(y)))$ :: value).

The element ( $x$ is (valid in $y)$ ) specifying that $x$ is valid in the context of variables $y$ is defined by the rule

(rule $(x$ is (valid in $y))$ var $(x)$ seq $(y)$ abn then $(x$ is (valid in $(y)))$ :: value).

The element ( $x$ is (sequence $y)$ ) specifying that $x$ is a sequence element such that the value in $\llbracket\left(e_{l}\right.$ is $\left.y\right) \rrbracket$ does not equal und for each element $e_{l}$ of $x$ is defined by the rule (rule $((x y)$ is (sequence $z))$ var $(x, z)$ seq $(y)$ abn 
then $((x$ is $z)$ and $((y)$ is (sequence $z)))$;

(rule $(()$ is (sequence $x)$ ) var $(x)$ abn then true).

The element ( $x$ is rule) specifying that $x$ is a rule is defined as follows:

(rule ( $x$ is rule) var $(x)$ abn then ( $x$ is rule) :: value);

(interpretation ( $x$ is rule) var $(x)$ then $f_{n}$ ),

where $\left[f_{n} s_{b}\right]=\left[\right.$ if $\left[x_{0} \in R_{l}\right]$ then true else und $]$.

The element ( $x$ is (rule in $y)$ ) specifying that $x$ is a rule in the context of the state $y$ is defined as follows:

(rule $(x$ is (rule in $y))$ var $(x, y)$ abn then $(x$ is (rule in $y))$ :: value);

(definition $(x$ is (rule in $y))$ var $(x, y)$ where $((x$ is rule) and $(y$ is state $))$

then ( $x$ is conceptual in $y)::$ atm);

(interpretation $(x$ is (conceptual in $y)$ ):: atm var $(x, y)$ then $\left.f_{n}\right)$,

where $\left[f_{n} s_{b}\right]=\left[\right.$ if $\left[x_{0} \in R_{l} \llbracket y_{0} \rrbracket\right]$ then true else und $]$.

The element ( $x$ is transition) specifying that $x$ is a transition is defined as follows:

(rule ( $x$ is transition) var $(x)$ abn then $(x$ is transition) :: value);

(interpretation ( $x$ is transition) var $(x)$ then $\left.f_{n}\right)$,

where $\left[f_{n} s_{b}\right]=\left[\right.$ if $\left[x_{0} \in T_{r n}\right]$ then true else und $]$.

\subsection{Elements operations}

The element () is defined by the rule

(rule () abn then ()::q).

The element (len $x$ ) specifying the length of the element $x$ is defined by the rule (rule (len $x)$ var $(x)$ val $(x)$ abn then $($ len $x:: *:: q)::$ value).

The element $(x=y)$ specifying the equality of the elements $x$ and $y$ is defined by the rule (rule $(x=y)$ var $(x, y)$ val $(x, y)$ abn then $(x:: *:: q=y:: *:: q)::$ value $)$.

The element $(x !=y)$ specifying the inequality of the elements $x$ and $y$ is defined in the similar way.

The element $(x \cdot y)$ specifying the $y$-th element of the sequence element $x$ is defined by the rule

(rule $(x . y)$ var $(x, y)$ val $(x, y)$ abn then $(x:: *:: q . y:: *:: q)::$ value $)$.

The element $(x \ldots y)$ specifying the value of the attribute element $x$ for the attribute $y$ is defined by the rule 
(rule $(x . . y) \operatorname{var}(x, y)$ val $(x)$ abn then $(x:: *:: q$.. y) :: value $)$.

The element $(x+y)$ specifying the concatenation of the sequence elements $x$ and $y$ is defined by the rule

(rule $(x+y) \operatorname{var}(x, y)$ val $(x, y)$ abn then $(x:: *:: q+y:: *:: q)::$ value $)$.

The element $(x+y)$ specifying the addition of the element $x$ to the head of the sequence element $y$ is defined by the rule

(rule $(x++y)$ var $(x, y)$ val $(x, y)$ abn then $(x:: *:: q .+y:: *:: q)::$ value $)$.

The element $(x+$. : set $y)$ specifying the addition of the element $x$ to the head of the sequence element $y$ representing a set is defined as follows:

(rule $(x+.:$ set $y)$ var $(x, y)$ val $(x, y)$ abn where $(y:: *$ is set $)$

then $(x:: *:: q$.+:: set $y:: *:: q)::$ value $)$.

The element $(x+. y)$ specifying the addition of the element $y$ to the tail of the sequence element $x$ is defined by the rule

(rule $(x+. y)$ var $(x, y)$ val $(x, y)$ abn then $(x:: *:: q+. y:: *:: q)::$ value $)$.

The element $(x+$.: set $y)$ specifying the addition of the element $y$ to the tail of the sequence element $x$ representing a set is defined by the rule

(rule $(x+.::$ set $y)$ var $(x, y)$ val $(x, y)$ abn where $(x:: *$ is set $)$

then $(x:: *:: q+$. $::$ set $y:: *:: q):: a b n)$.

The element $(x-$.:: set $y)$ specifying the deletion of the element $y$ from the sequence element $x$ representing a set is defined by the rule

(rule $(x-.::$ set $y)$ var $(x, y)$ val $(x, y)$ abn where $(x:: *$ is set $)$

then $(x:: *:: q-.::$ set $y:: *:: q)::$ value $)$.

The element (upd $x y_{1}: z_{1}, \ldots, y_{n_{t}}: z_{n_{t}}$ ) specifying the sequential updates of the attribute element $x$ at the points $y_{1}, \ldots, y_{n_{t}}$ by $z_{1}, \ldots, z_{n_{t}}$ is defined by the rules

(rule (upd $x$ y) var $(x)$ seq $(y)$ val $(x)$ abn

where $((x:: *$ is attribute) and $((y)$ is (sequence update $)))$ then (upd :: att $x:: * y))$;

(rule (upd :: att $x y$ z) var $(y)$ seq $(z)$ und $(x)$ abn

then (upd :: att (upd1 :: att $x$ y) z));

(rule (upd:: att $x$ ) var $(x)$ then $x)$;

(rule (upd1::att $x y: z)$ var $(x, y, z)$ val $(z)$ abn

then (upd1 :: att $x \quad y: z:: *:: q)::$ value). 
The element (upd $x y: z$ ) specifying the update of the sequence element $x$ at the index $y$ by $z$ is defined by the rule

(rule (upd $x y z) \operatorname{var}(x, y, z) \operatorname{val}(x, y, z)$ abn

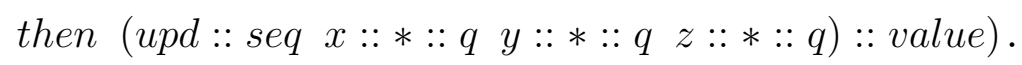

The element ( $x$ in $::$ set $y$ ) specifying that $x$ is an element of the sequence element $y$ is defined as follows:

(rule $(x$ in :: set $y)$ var $(x, y)$ val $(x, y)$ abn then $(x$ in :: set $y)::$ value $)$.

The element ( $x$ includes :: set $y$ ) specifying that the sequence element $x$ includes the elements of the sequence element $y$ is defined as follows:

(rule ( $x$ includes :: set $y)$ var $(x, y)$ val $(x, y)$ abn then ( $x$ includes :: set $y)$ :: value).

The element (attributes in $x$ ) specifying the sequence of attributes of the attribute element $x$ is defined by the rule

(rule (attributes in $x$ ) var (x) abn then (attributes in $x)$ :: value).

The element (values in $x$ ) specifying the sequence of attribute values of the attribute element $x$ is defined by the rule

(rule (values in $x$ ) var $(x)$ abn then (values in $x)::$ value).

The element (element in $x$ ) specifying the element of the sorted element $x$ is defined by the rule

(rule (element in $x)$ var $(x)$ abn then (element in $x)::$ value).

The element (sort in $x$ ) specifying the sort of the sorted element $x$ is defined by the rule (rule (sort in $x)$ var $(x)$ abn then (sort in $x)::$ value).

The element (attribute in $x$ ) specifying the attribute of the element update $x$ is defined by the rule

(rule (attribute in $x$ ) var (x) abn then (attribute in $x$ ) :: value).

The element (value in $x$ ) specifying the value of the element update $x$ is defined by the rule (rule (value in $x)$ var $(x)$ abn then (value in $x)::$ value).

The element (unbracket $(x)$ ) is defined by the rule (rule (unbracket $(x)$ ) seq $(x)$ abn then $x)$.

\subsection{Boolean operations}

The element true is defined by the rule:

(rule true abn then true :: value). 
The element ( $x$ and $y$ ) specifying the conjunction of $x$ and $y$ is defined by the rule: (rule ( $x$ and $y)$ var $(x, y)$ abn then (if $x$ then $y$ else und)).

The elements $\left(x o_{p} y\right)$, where $o_{p} \in\{o r,=>,<=>\}$ specifying the disjunction, implication and equivalence of $x$ and $y$ are defined in the similar way.

The element $\left(x_{1}\right.$ and $x_{2}$ and $\ldots$ and $\left.x_{n_{t}}\right)$ specifying the conjunction of $x_{1}, x_{2}, \ldots, x_{n_{t}}$ is defined by the rule

(rule $(x$ and $y$ and $z)$ var $(x, y) \operatorname{seq}(z)$ abn then $((x$ and $y)$ and $z)$.

The element $\left(x_{1}\right.$ or $x_{2}$ or $\ldots$ or $\left.x_{n_{t}}\right)$ specifying the disjunction of $x_{1}, x_{2}, \ldots, x_{n_{t}}$ is defined in the similar way.

The element $($ not $x$ ) specifying the negation of $x$ is defined by the rule (rule (not $x)$ var $(x)$ abn then (if $x$ then und else true)).

\subsection{Integers}

The element $i_{n t}$ is defined by the rule (rule $x$ var $(x)$ abn where $(x$ is int) then $x:: q)::$ name:: ("@", int).

The rule satisfies the property: ("@", exception) $\prec_{\llbracket o_{r d . t r n . e x} \rrbracket}(" @ "$, int $)$.

The element $(x+y)$ specifying the sum of $x$ and $y$ is defined by the rule (rule $(x+y)$ var $(x, y)$ val $(x, y)$ abn then $(x:: *:: q+y:: *:: q)::$ value $)$.

The elements $\left(x o_{p} y\right)$, where $o_{p} \in\{-, *$,div, $\bmod \}$, specifying the integer operations - , *, div and mod, are defined in the similar way.

The element $(x<y)$ specifying that $x$ is less than $y$ is defined by the rule (rule $(x<y)$ var $(x, y)$ val $(x, y)$ abn then $(x:: *:: q<y:: *:: q)::$ value $)$.

The elements $\left(x o_{p} y\right.$ ), where $o_{p} \in\{<=,>,>=\}$, specifying the integer relations $\leq,>$ and $\geq$, are defined in the similar way.

\subsection{Conceptuals operations}

The element ( $x$ in $y$ ) specifying the value of the conceptual $x$ in the state $y$ is defined by the rule

(rule $(x$ in $y)$ var $(x, y)$ abn then $(x$ in $y)::$ value $)$.

The element $x::$ state $:: y$ specifying the value of the conceptual $x$ in the substate with the name $y$ of the current configuration is defined by the rule (rule $x::$ state $:: y$ var $(x, y)$ abn then $(x::$ state $:: y)::$ value $)$. 
The element $c_{n c p l}$ is a shortcut for $c_{n c p l}::()$.

The assignment $\left(c_{n c p l}::\right.$ state $\left.:: n_{m}::=e_{l}\right)$ of $e_{l}$ to $c_{n c p l}::$ state $:: n_{m}$ is defined as follows: (rule $(x::$ state $:: z::=y)$ var $(x, y, z)$ val $(y)$ abn where ( $x$ is conceptual) then $(x::$ state $:: z::=y:: *)::$ atm); (transition $(x::$ state $:: z::=y):: \operatorname{atm} \operatorname{var}(x, y, z)$ then $\left.f_{n}\right)$, where $\left(x_{0}::\right.$ state $\left.:: z_{0}::=y_{0}\right)::$ atm, $e_{l . *} \# c_{n f} \rightarrow_{f_{n}, s_{b}} e_{l . *} \#\left[\left[c_{n f} z_{0}\right] x_{0}: y_{0}\right]$.

The element $\left(c_{n c p l}::=e_{l}\right)$ is a shortcut for $\left(c_{n c p l}::()::=e_{l}\right)$. The elements $\left(c_{n c p l}::\right.$ state :: $\left.n_{m}::=\right)$ and $\left(c_{n c p l}::=\right)$ are shortcuts for $\left(c_{n c p l}::\right.$ state $:: n_{m}::=$ und) and $\left(c_{n c p l}::=\right.$ und $)$.

\subsection{Countable concepts operations}

A normal element $c_{n c p . c}$ is a countable concept in $\llbracket c_{n f} \rrbracket$ if $\left[\left[c_{n f}\right.\right.$ countable-concept $]$ (0 : $\left.\left.c_{n c p . c}\right)\right] \in N_{t}$. Thus, the substate countable-concept specifies countable concepts. Let $C_{n c p . c}$ be a set of countable concepts. The element $\left[\left[c_{n f}\right.\right.$ countable-concept $\left.]\left(0: c_{n c p . c}\right)\right]$ is called an order in $\llbracket c_{n c p . c}, c_{n f} \rrbracket$. Let $O_{r d . c n c p . c}$ be a set of orders of countable concepts. An element $n_{t}:: c c:: c_{n c p . c}$ is called an instance in $\llbracket c_{n c p . c} \rrbracket$. An element $n_{t}:: c c:: c_{n c p . c}$ is an instance in $\llbracket c_{n c p . c}, c_{n f} \rrbracket$ if

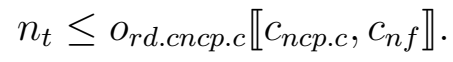

The element ( $x$ is countable-concept) specifying that $x$ is a countable concept is defined as follows:

(rule ( $x$ is countable-concept) var $(x)$ abn then ( $x$ is countable-concept) :: value).

The element $n_{t}:: c c:: c_{n c p . c}$ is defined by the rule: (rule $x:: c c:: y$ var $(x, y)$ abn then $x:: c c:: y::$ value).

Let $c_{n c p l}$ denote $(0: x)::$ countable-concept. The element (new $x$ ) called an instance generator generates a new instance of the countable concept $x$ and adds this concept if it was not. It is defined as follows:

(rule $($ new $x)$ var $(x)$ abn then $($ new $x)::$ atm);

(transition $($ new $x)$ :: atm var $(x)$ then $\left.f_{n}\right)$, where $\left(\right.$ new $\left.x_{0}\right)::$ atm, $e_{l . *} \# c_{n f} \rightarrow_{f_{n}, s_{b}}$ (let $w$ be $c_{n c p l}$ in (if ( $w$ is int) then (seq ( $c_{n c p l ~}:=$ $(w+1))$, (let $w 1$ be $(w+1)$ in $w 1:: x:: c c))$ else $\left.\left.\left(\operatorname{seq}\left(c_{n c p l}::=1\right), 1:: x:: c c\right)\right)\right)$, $e_{l . *} \# c_{n f}$.

\subsection{Matching operations}

The conditional pattern matching element $e_{l}$ of the form (if $x$ matches $y$ var z seq u then $v$ else $w)$, where $(y, z, u)$ is a pattern specification, is defined as follows: 
(rule (if $x$ matches $y$ var $z$ seq $u$ then $v$ else $w)$ var $(x, y, z, u)$ seq $(v, w)$ abn where ( $z$ is sequence) and ( $u$ is sequence) and ( $z$ includes $::$ set $u)$ )

then (if $x$ matches $y$ var z seq $u$ then $v$ else $w)::$ atm);

(transition (if $x$ matches $y$ var $z$ seq $u$ then $v$ else $w$ ) :: atm

$\operatorname{var}(x, y, z, u, v, w)$ then $\left.f_{n}\right)$,

where (if $x_{0}$ matches $y_{0}$ var $z_{0}$ seq $u_{0}$ then $v_{0}$ else $\left.w_{0}\right)::$ atm, $e_{l * *} \# c_{n f} \rightarrow_{f_{n}, s_{b}}\left[\right.$ if $\left[x_{0}\right.$ is an instance in $\llbracket\left(y_{0}, z_{0}, u_{0}\right), m_{t}, s_{b .1} \rrbracket$ for some $\left.s_{b .1}\right]$ then $\left[\right.$ subst $s_{b .1} \cup\left(\right.$ conf :: in : $c_{n f}$, val :: in : $\left.\left.v_{l} \llbracket c_{n f} \rrbracket\right) v_{0}\right]$ else $\left[\operatorname{subst}\left(\operatorname{conf}::\right.\right.$ in : $c_{n f}$, val :: in : $\left.\left.v_{l} \llbracket c_{n f} \rrbracket\right) w_{0}\right], e_{l . *} \# c_{n f}$. The objects $x, y$, $z, u, v$ and $w$ are called a matched element, pattern, variable specification, sequence variable specification, then-branch and else-branch in $\llbracket e_{l} \rrbracket$. The elements of $z$ are called pattern variables in $\llbracket e_{l} \rrbracket$. The element $e_{l}$ executes the instance of the then-branch $v$ in $\llbracket s_{b .1} \rrbracket$ if $x$ is an instance in $\llbracket y, s_{b .1} \rrbracket$. Otherwise, the element $e_{l}$ executes the else-branch $w$.

Let $\left\{v_{r . *}\right\},\left\{v_{r . s . *}\right\},\left\{v_{r . * 1}\right\}$ and $\left\{v_{r . * .2}\right\}$ are pairwise disjoint, and $\left\{v_{r . * .3}\right\} \subseteq\left\{v_{r . *}\right\} \cup\left\{v_{r . * .1}\right\} \cup$ $\left\{v_{r . * 2}\right\}$. The form (if $e_{l}$ matches $p_{t} \operatorname{var}\left(v_{r . *}\right) \operatorname{seq}\left(v_{r . s . *}\right)$ abn $\left(v_{r . * .1}\right)$ und $\left(v_{r . * 2}\right)$ val $\left(v_{r . * .3}\right)$ where $c_{n d}$ then $e_{l .1}$ else $e_{l .2}$ ) is defined as follows:

- $\left(\right.$ if $e_{l}$ matches $p_{t} \operatorname{var}\left(v_{r . *}\right) \operatorname{seq}\left(v_{r . s . *}\right)$ und $\left(v_{r . * .1}\right)$ abn $\left(v_{r . * .2}\right)$ val $\left(v_{r . * .3}\right)$ where $c_{n d}$ then $e_{l .1}$ else $\left.e_{l .2}\right)$ is a shortcut for (if $e_{l}$ matches $p_{t} \operatorname{var}\left(v_{r . *}\right) \operatorname{seq}\left(v_{r . s . *}\right)$ abn $\left(v_{r . * .1}\right)$ und $\left(v_{r . * .2}\right)$ val $\left(v_{r . * .3}\right)$ then (if $c_{n d}$ then $e_{l .1}$ else $e_{l .2}::$ (nosubstexcept conf :: in, val :: in)) else $\left.e_{l .2}\right)$;

- (if $e_{l}$ matches $p_{t} \operatorname{var}\left(v_{r . *}\right) \operatorname{seq}\left(v_{r . s . *}\right)$ und $\left(v_{r . * .1}\right)$ abn $\left(v_{r . * .2}\right)$ val $\left(v_{r . * .3}, v_{r}\right)$ then $e_{l .1}$ else $\left.e_{l .2}\right)$ is a shortcut for (if $e_{l}$ matches $p_{t} \operatorname{var}\left(v_{r . *}\right) \operatorname{seq}\left(v_{r . s . *}\right)$ und $\left(v_{r . * 1}\right)$ abn $\left(v_{r . * .2}\right)$ val $\left(v_{r . * .3}\right)$ then (let $w$ be $v_{r}$ in $\left.\left[\operatorname{subst}\left(v_{r}:: *: w\right) e_{l .1}\right]\right)$ else $\left.e_{l .2}\right)$, where $w$ is a new element that does not occur in this definition;

- (if $e_{l}$ matches $p_{t} \operatorname{var}\left(v_{r . *}\right) \operatorname{seq}\left(v_{r . s . *}\right)$ und $\left(v_{r . * .1}\right)$ abn $\left(v_{r . * .2}\right)$ val () then $e_{l .1}$ else $\left.e_{l .2}\right)$ is a shortcut for (if $e_{l}$ matches $p_{t}$ var $\left(v_{r . *}\right) \operatorname{seq}\left(v_{r . s . *}\right)$ und $\left(v_{r . * 1}\right)$ abn $\left(v_{r . * 2}\right)$ then $e_{l .1}$ else $\left.e_{l .2}\right)$

- (if $e_{l}$ matches $p_{t} \operatorname{var}\left(v_{r . *}\right) \operatorname{seq}\left(v_{r . s . *}\right)$ und $\left(v_{r . * .1}, v_{r}\right)$ abn $\left(v_{r . * 2}\right)$ then $\left.b_{d}\right)$ is a shortcut for (if $e_{l}$ matches $p_{t} \operatorname{var}\left(v_{r . *}\right) \operatorname{seq}\left(v_{r . s . *}\right)$ und $\left(v_{r . * .1}\right)$ abn $\left(v_{r . * .2}\right)$ then (if $\left(v_{r}\right.$ is undefined) then und else $e_{l .1}$ ) else $\left.e_{l .2}\right)$;

- (if $e_{l}$ matches $p_{t} \operatorname{var}\left(v_{r . *}\right) \operatorname{seq}\left(v_{r . s . *}\right)$ und () abn $\left(v_{r . * .2}\right)$ then $e_{l .1}$ else $\left.e_{l .2}\right)$ is a shortcut for (if $e_{l}$ matches $p_{t} \operatorname{var}\left(v_{r . *}\right) \operatorname{seq}\left(v_{r . s . *}\right)$ abn $\left(v_{r . * .2}\right)$ then $e_{l .1}$ else $\left.e_{l .2}\right)$;

- (if $e_{l}$ matches $p_{t} \operatorname{var}\left(v_{r . *}\right) \operatorname{seq}\left(v_{r . s . *}\right)$ abn $\left(v_{r . * 2}, v_{r}\right)$ then $e_{l .1}$ else $\left.e_{l .2}\right)$ is a shortcut for (if $e_{l}$ matches $p_{t} \operatorname{var}\left(v_{r . *}\right)$ seq $\left(v_{r . s . *}\right)$ abn $\left(v_{r . * 2}\right)$ then (if $\left(v_{r}\right.$ is abnormal) then $v_{r}$ else 
$\left.e_{l .1}\right)$ else $\left.e_{l .2}\right)$;

- (if $e_{l}$ matches $p_{t} \operatorname{var}\left(v_{r . *}\right)$ seq $\left(v_{r . s . *}\right)$ abn () then $e_{l .1}$ else $\left.e_{l .2}\right)$ is a shortcut for (if $e_{l}$ matches $p_{t} \operatorname{var}\left(v_{r . *}\right) \operatorname{seq}\left(v_{r . s . *}\right)$ then $e_{l .1}$ else $\left.e_{l .2}\right)$.

The element $c_{n d}$ specifies the restriction on the values of the pattern variables. The undefined value is propagated through the variables of $v_{r . * .1}$. Abnormal values are propagated through the variables of $v_{r . * 2}$. The special element $v_{r}:: *$ references to the value of element associated with the pattern variable $v_{r}$. A pattern variable is evaluated if the element associated with it is evaluated. Thus, the sequence $v_{r . * 3}$ contains evaluated pattern variables. A pattern variable is quoted if the element associated with it is not evaluated.

The objects $\operatorname{var}\left(v_{r . *}\right)$, seq $\left(v_{r . s . *}\right)$, und $\left(v_{r . * .1}\right)$, abn $\left(v_{r . * .2}\right)$, val $\left(v_{r . * .3}\right)$, where $c_{n d}$ and else $e_{l .2}$ in this form can be omitted. The omitted objects correspond to var (), seq (), und (), abn (), val (), where true and else skip, respectively.

The form $\left(e_{l}\right.$ matches $p_{t} \operatorname{var}\left(v_{r . *}\right) \operatorname{seq}\left(v_{r . s . *}\right)$ und $\left(v_{r . * .1}\right)$ abn $\left(v_{r . * .2}\right)$ val $\left(v_{r . * .3}\right)$ where $\left.c_{n d}\right)$ is a shortcut for $\left(\right.$ if $e_{l}$ matches $p_{t}$ var $\left(v_{r . *}\right) \operatorname{seq}\left(v_{r . s . *}\right)$ und $\left(v_{r . * 1}\right)$ abn $\left(v_{r . * 2}\right)$ val $\left(v_{r . * 3}\right)$ where $c_{n d}$ then true else und). The objects $\operatorname{var}\left(v_{r . *}\right)$, seq $\left(v_{r . s . *}\right)$, und $\left(v_{r . * .1}\right)$, abn $\left(v_{r . * .2}\right)$, val $\left(v_{r . * .3}\right)$ and where $c_{n d}$ in this form can be omitted. The omitted objects correspond to var (), seq (), und (), abn (), val () and where true, respectively.

\subsection{Interpretations operations}

The element ( $x$ is definition-form) specifying that $x$ is a definition form is defined as follows:

(rule ( $x$ is definition-form) var $(x)$ abn then ( $x$ is definition-form) :: value);

(transition ( $x$ is definition-form) var $(x)$ then $\left.f_{n}\right)$,

where $\left[f_{n} s_{b}\right]=\left[\right.$ if $\left[x_{0} \in F_{r m . d}\right]$ then true else und $]$.

The element $f_{r m . d}::$ name $:: n_{m}$ specifying a definition with the name $n_{m}$ is defined as follows:

(rule $x::$ name :: $y$ var $(x, y)$ abn where ( $x$ is definition-form)

then $x::$ name :: $y::$ atm $::$ definition);

(transition $x::$ name $:: y::$ atm $::$ definition var $(x, y)$ then $\left.f_{n}\right)$, where

- if $y_{0} \in\left[\right.$ support $\left[c_{n f}(0:\right.$ definitions $)::$ state $::$ interpretation $\left.]\right] \cup\left[\right.$ support $\left.i_{n t r . a . s}\right]$, then $x_{0}::$ name $:: y_{0}::$ atm $::$ definition, $e_{l . *} \# c_{n f} \rightarrow_{f_{n}, s_{b}} e_{l . *} \#$ und $\# c_{n f}$; 
- if $y_{0} \notin\left[\right.$ support $\left[c_{n f}(0:\right.$ definitions $)::$ state $::$ interpretation $\left.]\right] \cup\left[\right.$ support $\left.i_{n t r . a . s}\right]$, and $x_{0}$ is reduced to $d_{f}$, then $x_{0}::$ name $:: y_{0}::$ atm $::$ definition, $e_{l . *} \# c_{n f} \rightarrow_{f_{n}, s_{b}}$ $e_{l . *} \#\left[c_{n f}\right.$ interpretation. $(0:$ definitions $\left.) . y_{0}: d_{f}\right]$.

The element (add-interpretation $x$ ) adding the interpretation with the name $x$ is defined as follows:

(rule (add-interpretation $x)$ var $(x)$ abn then (add-interpretation $x)::$ atm); (transition (add-interpretation $x)::$ atm var $(x)$ then $\left.f_{n}\right)$, where

- if $x_{0} \in\left[\right.$ support $\left[c_{n f}(0:\right.$ definitions $)::$ state $::$ interpretation $\left.]\right] \cup\left[\right.$ support $\left.i_{n t r . a . s}\right]$, then $\left(\right.$ add-interpretation $\left.x_{0}\right)::$ atm,$e_{l . *} \# c_{n f} \rightarrow_{f_{n}, s_{b}} e_{l . *} \#\left[c_{n f}\right.$ interpretation.(0 : order $)$ : $\left[\right.$ value $\left[\left[c_{n f}(0:\right.\right.$ order $)::$ state $::$ interpretation $]:: q+$.: set $\left.\left.\left.x_{0}:: q\right] c_{n f}\right]\right]$;

- if $x_{0} \notin\left[\right.$ support $\left[c_{n f}(0:\right.$ definitions $)::$ state $::$ interpretation $\left.]\right] \cup\left[\right.$ support $\left.i_{n t r . a . s}\right]$, then $\left(\right.$ add-interpretation $\left.x_{0}\right)::$ atm, $e_{l . *} \# c_{n f} \rightarrow_{f_{n}, s_{b}} e_{l . *} \#$ und $\# c_{n f}$.

The element (add-interpretation $x$ after $y$ ) adding the interpretation with the name $x$ after the interpretation with the name $y$ is defined as follows:

(rule (add-interpretation $x$ after $y)$ var $(x, y)$ abn

then (add-interpretation $x$ after $y)::$ atm);

(transition (add-interpretation $x$ after $y)::$ atm var $(x, y)$ then $\left.f_{n}\right)$, where

- if $x_{0} \in\left[\right.$ support $\left[c_{n f}(0:\right.$ definitions $)::$ state $::$ interpretation $\left.]\right] \cup\left[\right.$ support $\left.i_{n t r . a . s}\right]$, and $y_{0} \notin\left[c_{n f}(0:\right.$ order $)::$ state $::$ interpretation $]:: q-$ - :: set $\left.x_{0}\right]$, then (add-interpretation $\left.x_{0}\right):: a t m, e_{l . *} \# c_{n f} \rightarrow f_{n}, s_{b} e_{l . *} \#$ und $\# c_{n f}$;

- if $x_{0} \in\left[\right.$ support $\left[c_{n f}(0:\right.$ definitions $)::$ state $::$ interpretation $\left.]\right] \cup\left[\right.$ support $\left.i_{n t r . a . s}\right]$, and $\left[\right.$ value $\left[c_{n f}(0:\right.$ order $)::$ state $::$ interpretation $]:: q-$ - : set $\left.x_{0}\right]=n_{m . * .1} y_{0} n_{m . * .2}$, then $\left(\right.$ add-interpretation $\left.x_{0}\right)::$ atm, $e_{l . *} \# c_{n f} \rightarrow_{f_{n}, s_{b}} e_{l . *} \#\left[c_{n f}\right.$ interpretation.(0 : order $)$ : $\left.n_{m . * .1} y_{0} x_{0} n_{m . * 2}\right]$;

- if $x_{0} \notin\left[\right.$ support $\left[c_{n f}(0:\right.$ definitions $)::$ state $::$ interpretation $\left.]\right] \cup\left[\right.$ support $\left.i_{n t r . a . s}\right]$, then $\left(\right.$ add-interpretation $\left.x_{0}\right)::$ atm, $e_{l . *} \# c_{n f} \rightarrow_{f_{n}, s_{b}} e_{l . *} \#$ und $\# c_{n f}$.

The element (delete-interpretation $x$ ) deleting the interpretation with the name $x$ is defined as follows:

(rule (delete-interpretation $x$ ) var $(x)$ abn then (delete-interpretation $x$ ) :: atm); (transition (delete-interpretation $x)$ :: atm var $(x)$ then $\left.f_{n}\right)$, 
where

- if $x_{0} \in\left[\right.$ support $\left[c_{n f}(0:\right.$ definitions $)::$ state $::$ interpretation $\left.]\right] \cup\left[\right.$ support $\left.i_{n t r . a . s}\right]$, then (delete-interpretation $\left.x_{0}\right)::$ atm, $e_{l . *} \# c_{n f} \rightarrow_{f_{n}, s_{b}} e_{l . *} \#\left[c_{n f}\right.$ interpretation.(0: order $):\left[\right.$ value $\left[c_{n f}(0:\right.$ order $)::$ state $::$ transition $]:: q-$ - :: set $\left.\left.x_{0}:: q c_{n f}\right]\right]$;

- if $x_{0} \notin\left[\right.$ support $\left[c_{n f}(0:\right.$ definitions $)::$ state $::$ interpretation $\left.]\right] \cup\left[\right.$ support $\left.i_{n t r . a . s}\right]$, then $\left(\right.$ delete-interpretation $\left.x_{0}\right)::$ atm, $e_{l . *} \# c_{n f} \rightarrow_{f_{n}, s_{b}} e_{l . *} \#$ und $\# c_{n f}$.

\subsection{Configurations operations}

The element conf :: cur specifying the current configuration is defined as follows: (rule conf :: cur abn then conf :: cur :: value).

The element val :: cur specifying the value in the current configuration is defined as follows: (rule val :: cur abn then val :: cur :: value); (definition val :: cur then val :: cur :: value); (interpretation val :: cur then $f_{n}$ ), where $\left[f_{n} s_{b}\right]=v_{l} \llbracket c_{n f} \rrbracket$.

\subsection{Transitions operations}

The element ( $x$ is rule-form) specifying that $x$ is a rule form is defined as follows: (rule $(x$ is rule-form) var $(x)$ abn then $(x$ is rule-form) :: value); (transition ( $x$ is rule-form) var $(x)$ then $\left.f_{n}\right)$, where $\left[f_{n} s_{b}\right]=\left[\right.$ if $\left[x_{0} \in F_{r m . r}\right]$ then true else und $]$.

The element $f_{r m . r}::$ name $:: n_{m}$ specifying a rule with the name $n_{m}$ is defined as follows: (rule $x::$ name :: $y$ var $(x, y)$ abn where $(x$ is rule-form)

then $x::$ name :: $y::$ atm $::$ rule);

(transition $x::$ name $:: y::$ atm :: rule var $(x, y)$ then $\left.f_{n}\right)$, where

- if $y_{0} \in\left[\right.$ support $\left[c_{n f}(0:\right.$ rules $)::$ state $::$ transition $\left.]\right] \cup\left[\right.$ support $\left.t_{\text {rn.rlt.ex.s }}\right] \cup[$ support $\left.t_{\text {rn.rlt.en.s }}\right]$, then $x_{0}::$ name $:: y_{0}::$ atm :: rule, $e_{l . *} \# c_{n f} \rightarrow_{f_{n}, s_{b}} e_{l . *} \#$ und \# $c_{n f}$;

- if $y_{0} \notin\left[\right.$ support $\left[c_{n f}(0:\right.$ rules $)::$ state :: transition $\left.]\right] \cup\left[\right.$ support $\left.t_{\text {rn.rlt.ex.s }}\right] \cup[$ support $\left.t_{r n . r l t . e n . s}\right]$, and $x_{0}$ is reduced to $r_{l}$, then $x_{0}::$ name $:: y_{0}::$ atm $::$ rule, $e_{l . *} \# c_{n f} \rightarrow_{f_{n}, s_{b}}$ $e_{l . *} \#\left[c_{n f}\right.$ transition. $(0:$ rules $\left.) . y_{0}: r_{l}\right]$.

The element (add-transition $x$ ) adding the transition with the name $x$ is defined as follows: 
(rule (add-transition $x$ ) var $(x)$ abn then (add-transition $x$ ) :: atm);

(transition (add-transition $x)::$ atm var $(x)$ then $\left.f_{n}\right)$,

where

- if $x_{0} \in\left[\right.$ support $\left[c_{n f}(0:\right.$ rules $)::$ state $::$ transition $\left.]\right] \cup\left[\right.$ support $\left.t_{\text {rn.rlt.ex.s }}\right]$, then $\left(\right.$ add-transition $\left.x_{0}\right)::$ atm, $e_{l . *} \# c_{n f} \rightarrow_{f_{n}, s_{b}} e_{l . *} \#\left[c_{n f}\right.$ transition. $(-1$ : exogenous, 0 : order $):\left[\right.$ value $\left[\left[c_{n f}(-1:\right.\right.$ exogenous, $0:$ order $)::$ state $::$ transition $]:: q+$ +: set $x_{0}::$ q] $\left.\left.c_{n f}\right]\right]$;

- if $x_{0} \in\left[\right.$ support $\left.t_{r n . r l t . e n . s}\right]$, then (add-transition $\left.x_{0}\right):: a t m, e_{l . *} \# c_{n f} \rightarrow_{f_{n}, s_{b}} e_{l . *} \#\left[c_{n f}\right.$ transition. $(-1$ : endogenous, 0 : order $):\left[\right.$ value $\left[\left[c_{n f}(-1\right.\right.$ : endogenous, 0 : order $)::$ state $::$ transition $]:: q+$. :: set $\left.\left.\left.x_{0}:: q\right] c_{n f}\right]\right]$;

- if $x_{0} \notin\left[\right.$ support $\left[c_{n f}(0:\right.$ rules $)::$ state :: transition $\left.]\right] \cup\left[\right.$ support $\left.t_{\text {rn.rlt.ex.s }}\right] \cup[$ support $\left.t_{\text {rn.rlt.en.s }}\right]$, then (add-transition $\left.x_{0}\right)::$ atm, $e_{l . *} \# c_{n f} \rightarrow_{f_{n}, s_{b}} e_{l . *} \#$ und $\# c_{n f}$.

The element (add-transition $x$ after $y$ ) adding the transition with the name $x$ after the transition with the name $y$ is defined as follows:

(rule (add-transition $x$ after $y)$ var $(x, y)$ abn then (add-transition $x$ after $y)::$ atm); (transition (add-transition $x$ after $y):: \operatorname{atm} \operatorname{var}(x, y)$ then $\left.f_{n}\right)$, where

- if $x_{0} \in\left[\right.$ support $\left[c_{n f}(0:\right.$ rules $)::$ state :: transition $\left.]\right] \cup\left[\right.$ support $\left.t_{\text {rn.rlt.ex.s }}\right]$, and $y_{0} \notin$ $\left[c_{n f}(-1:\right.$ exogenous, $0:$ order $)::$ state $::$ transition $]:: q-$ - :: set $\left.x_{0}\right]$, then (addtransition $\left.x_{0}\right)::$ atm, $e_{l . *} \# c_{n f} \rightarrow_{f_{n}, s_{b}} e_{l . *} \#$ und \# $c_{n f}$;

- if $x_{0} \in\left[\right.$ support $\left[c_{n f}(0:\right.$ rules $)::$ state :: transition $\left.]\right] \cup\left[\right.$ support $\left.t_{\text {rn.rlt.ex.s }}\right]$, and $\left[\right.$ value $\left[c_{n f}\right.$ $(-1:$ exogenous, $0:$ order $)::$ state $::$ transition $]:: q-$ - :: set $\left.x_{0}\right]=n_{m . * .1} y_{0} n_{m . * .2}$, then $\left(\right.$ add-transition $\left.x_{0}\right)::$ atm, $e_{l . *} \# c_{n f} \rightarrow_{f_{n}, s_{b}} e_{l . *} \#\left[c_{n f}\right.$ transition. $(-1$ : exogenous, 0 : order) : $\left.n_{m . * .1} y_{0} x_{0} n_{m . * 2}\right]$;

- if $x_{0} \in\left[\right.$ support $\left.t_{\text {rn.rlt.en.s }}\right]$, and $y_{0} \notin\left[c_{n f}(-1:\right.$ endogenous, 0 : order $)::$ state :: transition] $:: q-$ - : set $\left.x_{0}\right]$, then $\left(\right.$ add-transition $\left.x_{0}\right)::$ atm, $e_{l . *} \# c_{n f} \rightarrow_{f_{n}, s_{b}}$ $e_{l . *} \#$ und $\# c_{n f}$

- if $x_{0} \in\left[\right.$ support $\left.t_{\text {rn.rlt.en.s }}\right]$, and [value $\left[c_{n f}(-1:\right.$ endogenous, 0 : order $)::$ state :: transition $]:: q-.::$ set $\left.x_{0}\right]=n_{m . * .1} y_{0} n_{m . * .2}$, then $\left(\right.$ add-transition $\left.x_{0}\right)::$ atm, $e_{l . *} \# c_{n f}$ $\rightarrow_{f_{n}, s_{b}} e_{l . *} \#\left[c_{n f}\right.$ transition.(-1 : endogenous, $0:$ order $\left.): n_{m . * .1} y_{0} x_{0} n_{m . * .2}\right]$;

- if $x_{0} \notin\left[\right.$ support $\left[c_{n f}(0:\right.$ rules $)::$ state :: transition $\left.]\right] \cup\left[\right.$ support $\left.t_{\text {rn.rlt.ex.s }}\right] \cup[$ support 
$\left.t_{r n . r l t . e n . s}\right]$, then (add-transition $\left.x_{0}\right)::$ atm, $e_{l . *} \# c_{n f} \rightarrow_{f_{n}, s_{b}} e_{l . *} \#$ und $\# c_{n f}$.

The element (delete-transition $x$ ) deleting the transition with the name $x$ is defined as follows:

(rule (delete-transition $x$ ) var $(x)$ abn then (delete-transition $x$ ) :: atm);

(transition (delete-transition $x)::$ atm var $(x)$ then $f_{n}$ ), where

- if $x_{0} \in\left[\right.$ support $\left[c_{n f}(0:\right.$ rules $)::$ state :: transition $\left.]\right] \cup\left[\right.$ support $\left.t_{r n . r l t . e x . s}\right]$, then $\left(\right.$ delete-transition $\left.x_{0}\right)::$ atm, $e_{l . *} \# c_{n f} \rightarrow_{f_{n}, s_{b}} e_{l . *} \#\left[c_{n f}\right.$ transition.( -1 : exogenous, 0 : order $):\left[\right.$ value $\left[c_{n f}(-1:\right.$ exogenous, $0:$ order $)::$ state $::$ transition $]:: q-$. : set $x_{0}::$ $\left.\left.q c_{n f}\right]\right]$;

- if $x_{0} \in\left[\right.$ support $\left.t_{r n . r l t . e n . s}\right]$, then $\left(\right.$ delete-transition $\left.x_{0}\right)::$ atm, $e_{l . *} \# c_{n f} \rightarrow_{f_{n}, s_{b}} e_{l . *} \#\left[c_{n f}\right.$ transition. $(-1$ : endogenous, 0 : order $):\left[\right.$ value $\left[c_{n f}(-1\right.$ : endogenous, 0 : order $)::$ state $::$ transition $]:: q-$ - : set $\left.\left.x_{0}:: q c_{n f}\right]\right]$;

- if $x_{0} \notin\left[\right.$ support $\left[c_{n f}(0\right.$ : rules $)::$ state :: transition $\left.]\right] \cup\left[\right.$ support $\left.t_{\text {rn.rlt.ex.s }}\right] \cup[$ support $\left.t_{\text {rn.rlt.en.s }}\right]$, then (delete-transition $\left.x_{0}\right)::$ atm, $e_{l . *} \# c_{n f} \rightarrow_{f_{n}, s_{b}} e_{l . *} \#$ und $\# c_{n f}$.

The element $e_{l}$ of the form (modify $x$ ) or (modify $\left.:: n x\right)$ is defined as follows:

(rule (modify $x)$ var $(x)$ then (modify $x)::$ atm);

(rule (modify:: $n x)$ var $(x)$ abn then (modify $x)::$ atm);

(transition (modify $x$ ) :: atm var $(x)$ then $\left.f_{n}\right)$,

where $\left(\operatorname{modify} x_{0}\right)::$ atm, $e_{l . *} \# v_{l} \# c_{n f} \rightarrow_{f_{n}, s_{b}} e_{l . *} \#\left[\right.$ if [there exists $c_{n f .1}$ such that [value $\left[\right.$ subst $\left(\right.$ conf :: in : $c_{n f}$, val :: in : $v_{l} \llbracket c_{n f} \rrbracket$, conf :: out : $c_{n f .1}$, val :: out : $\left.\left.\left.v_{l} \llbracket c_{n f .1} \rrbracket\right) x_{0}\right] c_{n f}\right] \neq$ und then $v_{l} \# c_{n f .1}$ else und \# $\left.c_{n f}\right]$. The element $x$ is called a transition condition in $\llbracket e_{l} \rrbracket$. It specifies the set of configurations reachable from $c_{n f}$ for one transition. The elements conf :: in and conf :: out reference to the input state and the output state, and the elements val :: in and val :: out reference to values in these states.

$\bigoplus$ The execution of the element (modify $(((-1:$ value, $0: x, 1:$ variable $)$ inconf $::$ out $)=$ $0)$ ) initiates the transition to a state in which the value of the variable $x$ equals to 0 .

$\bigoplus$ The execution of the element (modify $(((-1:$ value, $0: x, 1:$ variable $)=$ "green") and $(((-1:$ value, $0: x, 1:$ variable $)$ in conf $::$ out $)=$ "red" $)))$ initiates the transition from a state in which the value of the variable $x$ equals to "green" to a state in which the variable $x$ equals to "red". 
The element $e_{l}$ of the form (modify-exist $\left.(x) y\right)$ or (modify-exist :: $\left.n(x) y\right)$ is defined as follows:

(rule (modify-exist $(x) y)$ var $(y)$ seq $(x)$ then (modify-exist $(x) y)$ :: atm); (rule (modify-exist :: $n(x) y)$ var $(y) \operatorname{seq}(x)$ abn then (modify-exist $(x) y):: a t m)$; (transition (modify-exist $(x) y):: \operatorname{atm} \operatorname{var}(y)$ seq $(x)$ then $\left.f_{n}\right)$, where (modify-exist $\left.\left(x_{0}\right) y_{0}\right):: a t m, e_{l . *} \# v_{l} \# c_{n f} \rightarrow_{f_{n}, s_{b}} e_{l . *} \#\left[\right.$ if [there exists $c_{n f .1}$ such that $\left[\left[\operatorname{subst}\left(\operatorname{conf}::\right.\right.\right.$ in $: c_{n f}$, val :: in : $v_{l} \llbracket c_{n f} \rrbracket$, conf $::$ out $: c_{n f .1}$, val :: out : $\left.\left.v_{l} \llbracket c_{n f .1} \rrbracket\right) y_{0}\right]$ is satisfiable in $\left.\left(\left(x_{0}\right), c_{n f}\right)\right]$ then $v_{l} \# c_{n f .1}$ else und \# $\left.c_{n f}\right]$. The element $y$ is called a transition condition in $\llbracket e_{l} \rrbracket$. The elements of $x$ are called existential variables in $\llbracket e_{l} \rrbracket$.

\subsection{Safety operations}

The element $e_{l}$ of the form (assert $x$ ) or (assert $:: n x$ ) is defined as follows:

(rule (assert $x)$ var $(x)$ then (assert $x)::$ atm);

(rule (assert $x:: n)$ var $(x)$ abn then (assert $x)::$ atm);

(transition (assert $x$ ):: atm var $(x)$ then $\left.f_{n}\right)$,

where $\left(\right.$ assert $\left.x_{0}\right)::$ atm, $e_{l . *} \# v_{l} \# c_{n f} \rightarrow_{f_{n}, s_{b}} e_{l . *} \#\left[\right.$ if [[value [subst (conf :: inc $c_{n f}$, val :: in : $\left.\left.\left.v_{l}\right) x_{0}\right] c_{n f} \rrbracket \neq u n d\right]$ then $v_{l}$ else und $\# c_{n f}$. The element $x$ is called a safety condition in $\llbracket e_{l} \rrbracket$.

\subsection{Branching operations}

The element $e_{l}$ of the form (branching $x$ ) is defined as follows:

(rule (branching $x)$ seq $(x)$ abn then (branching $x)$ :: atm);

(transition (branching $x)$ :: atm var $(x)$ then $\left.f_{n}\right)$,

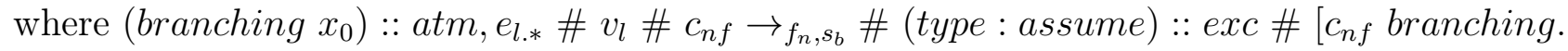
$(0:()):\left[\left(\left(x_{0}\right), c_{n f},\left(e_{l . *}\right)\right) .+\left[\left[c_{n f}\right.\right.\right.$ branching $\left.\left.\left.](0:())\right]\right]\right]$. The elements of $x$ are called branches in $\llbracket e_{l} \rrbracket$. The element $e_{l}$ generates the branchpoint with the branches $x$. The exception (type : assume) :: exc specifies the failure of the execution of the current branch. The substate branching contains information about branching. The conceptual $(0:())::$ state :: branching specifies the current sequence of branchpoints.

The endogenous transition relation specifying branching is defined as follows: (endogenous-transition $\left.f_{n}\right)$ :: name :: branching where

- if $\left[\left[c_{n f}\right.\right.$ branching $\left.](0:())\right]=\left(\left(\left(e_{l . * 1}, e_{l}, e_{l . * .2}\right), c_{n f .1},\left(e_{l * * .3}\right)\right), e_{l . *}\right)$, then \# (type : 


$$
\begin{aligned}
& \text { assume }):: \text { exc } \# c_{n f} \rightarrow_{\text {branching }} e_{l . * .3} \#\left[c_{n f .1} \text { branching. }(0:()):\left(\left(\left(e_{l . * .1}, e_{l . * 2}\right), c_{n f .1}\right.\right. \text {, }\right. \\
& \left.\left.\left.\left(e_{l . * .3}\right)\right), e_{l . *}\right)\right]
\end{aligned}
$$

- if $\left[\left[c_{n f}\right.\right.$ branching $\left.](0:())\right]=\left(\left((), c_{n f .1},\left(e_{l * * 3}\right)\right), e_{l . *}\right)$, then \# (type : assume) ::

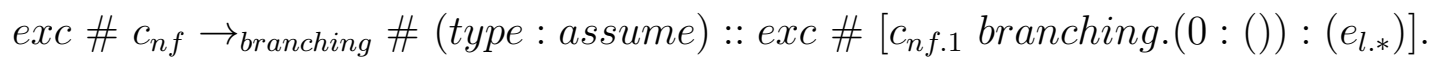

The element $e_{l}$ of the form (assume $x$ ) or (assume :: $n x$ ) is defined as follows:

(rule (assume $x)$ var $(x)$ then (assume $x)$ :: atm);

(rule (assume :: $n x)$ var $(x)$ abn then (assume $x)::$ atm);

(transition (assume $x$ ) :: atm var $(x)$ then $\left.f_{n}\right)$,

where $\left(\right.$ assume $\left.x_{0}\right)::$ atm, $e_{l * *} \# v_{l} \# c_{n f} \rightarrow_{f_{n}, s_{b}}\left[\right.$ if [[value [subst (conf :: in : $c_{n f}$, val :: in : $\left.\left.\left.\left.v_{l} \llbracket c_{n f} \rrbracket\right) x_{0}\right] c_{n f}\right] \neq u n d\right]$ then $e_{l . *} \# v_{l}$ else \# (type : assume) :: exc] \# $c_{n f}$. The element $x$ is called a continuation condition in $\llbracket e_{l} \rrbracket$. The violation of this condition initiates the failure of the execution of the current branch.

The element $e_{l}$ of the form (assume-exist $\left.(x) y\right)$ or (assume-exist :: $\left.n(x) y\right)$ is defined as follows:

(rule (assume-exist $(x) y)$ var $(y)$ seq $(x)$ then (assume-exist $x)$ :: atm);

(rule (assume-exist :: $n(x) y)$ var $(y)$ seq $(x)$ abn then (assume-exist $x)::$ atm);

(transition (assume-exist $(x) y)$ :: atm var $(y)$ seq $(x)$ then $\left.f_{n}\right)$,

where (assume $\left.\left(x_{0}\right) y_{0}\right)::$ atm, $e_{l * *} \# v_{l} \# c_{n f} \rightarrow_{f_{n}, s_{b}}\left[\right.$ if $\left[\left[\operatorname{subst}\right.\right.$ (conf :: in : $c_{n f}$, val :: in : $\left.v_{l} \llbracket c_{n f} \rrbracket\right) y_{0} \rrbracket$ is satisfiable in $\left.\llbracket\left(x_{0}\right), c_{n f} \rrbracket\right]$ then $e_{l . *} \# v_{l}$ else \# (type : assume) :: exc \# \# $c_{n f}$. The element $y$ is called a continuation condition in $\llbracket e_{l} \rrbracket$. The elements of $x$ are called existential variables in $\llbracket e_{l} \rrbracket$.

\section{Justification of requirements for conceptual transition systems}

In this section, we establish that CTSs meet the additional requirements stated in section 1:

8. The formalism must have language support. The language associated with the formalism must define syntactic representations of models of states, state objects, queries, query objects, answers and answer objects and includes the set of predefined basic query models. The CTSL language associated with CTSs defines syntactic representations of models of states, state objects, queries, query objects, answers and answer objects and includes the set of predefined basic query models.

9. It must model the change of the conceptual structure of states and state objects of the ITS. The change of the conceptual structure of the ITS is described by the transition relation 
on conceptual configurations specifying conceptual structures of the ITS with different sets of ontological elements.

10. It must model the change of the content of the conceptual structure. The change of the content of the conceptual structure of the ITS is described by the transition relation on conceptual states specifying the same conceptual structure of the ITS. In fact, the distinction between requirements 9 and 10 is relative, for conceptuals allow to define classifications of ontological elements with different granularity.

11. It must model the transition relations of the ITS. The transition relations of the ITS are modelled by the transition relation $t_{r n . r l t}$ of the CTS.

12. The model of the exogenous transition relation must be extensible. The model of the exogenous transition relation of the IQS is extended by addition of trnasition rules.

Thus, the additional requirements are met for CTSs.

\section{Conclusion}

In the paper two formalisms (ITSs and CTSs) for abstract unified modelling of the artifacts of the conceptual design of information systems have been proposed by ontological elements with arbitrary conceptual granularity. The basic definitions of the theory of CTSs have been given. The language of CTSs has been defined.

We plan to use CTSs to design and prototype software systems as well as to specify operational and axiomatic semantics of programming languages. In the case of operational semantics of a programming language, CTSs model an abstract machine of the language. In the case of axiomatic semantics of a programming language, CTSs model a verification conditions generator for programs in the language.

\section{References}

1. Sokolowski J., Banks C. Modeling and Simulation Fundamentals: Theoretical Underpinnings and Practical Domains. Wiley, 2010.

2. Chen P. Entity-relationship modeling: historical events, future trends, and lessons learned // Software pioneers. Springer-Verlag New York, 2002. P. 296-310.

3. Anureev I.S. Formalisms for conceptual design of closed information systems // System Informatics. 2016. N 7. P. 69-148.

4. Gurevich Y. Abstract state machines: An Overview of the Project // Foundations of Information and Knowledge Systems. Lect. Notes Comput. Sci. 2004. Vol. 2942. P. 6-13. 
5. Gurevich Y. Evolving Algebras. Lipari Guide // Specification and Validation Methods. Oxford University Press, 1995. P. 9-36. 
